\title{
Simulation-based uncertainty quantification of human arterial network hemodynamics
}

\author{
Peng Chen ${ }^{1, *}$, Alfio Quarteroni ${ }^{1,2}$, Gianluigi Rozza ${ }^{3}$ \\ ${ }^{1}$ Modelling and Scientific Computing, CMCS, Mathematics Institute of Computational Science and Engineering, \\ MATHICSE, Ecole Polytechnique Fédérale de Lausanne, EPFL, Station 8, CH-1015 Lausanne, Switzerland. \\ ${ }^{2}$ Modellistica e Calcolo Scientifico, MOX, Dipartimento di Matematica F. Brioschi, Politecnico di Milano, P.za \\ Leonardo da Vinci 32, I-20133, Milano, Italy. \\ ${ }^{3}$ SISSA MathLab, International School for Advanced Studies, via Bonomea 265, 34136 Trieste, Italy.
}

\begin{abstract}
SUMMARY
This work aims at identifying and quantifying uncertainties from various sources in human cardiovascular system based on stochastic simulation of a one dimensional arterial network. A general analysis of different uncertainties and probability characterization with log-normal distribution of these uncertainties is introduced. Deriving from a deterministic one dimensional fluid structure interaction model, we establish the stochastic model as a coupled hyperbolic system incorporated with parametric uncertainties to describe the blood flow and pressure wave propagation in the arterial network. By applying a stochastic collocation method with sparse grid technique, we study systemically the statistics and sensitivity of the solution with respect to many different uncertainties in a relatively complete arterial network with potential physiological and pathological implications for the first time. Copyright (c) 2013 John Wiley \& Sons, Ltd.
\end{abstract}

Received ...

KEY WORDS: uncertainty quantification, sensitivity analysis, stochastic collocation method, cardiovascular modelling, human arterial network, wave propagation, hemodynamics

\section{INTRODUCTION}

Mathematical modelling and numerical simulation of human cardiovascular system have undergone a vast development over the last few decades thanks to better understanding of the morphology and functionality of cardiovascular system, the availability of abundant clinical data as well as fast growing of computational resources [38, 23]. Specifically, various deterministic models targeted for the full human arterial tree, or specific sites (e.g. the carotid bifurcation, the aortic arch etc.), have been established [23, 2]. For instance, Navier-Stokes equations and elastic or viscoelastic equations are coupled together to characterize the fluid structure interaction property between the blood flow and the arterial wall in three dimensional configurations [23]; the one dimensional hyperbolic system simplified from the full three dimensional equations together with appropriate coupling conditions at the vascular junctions are widely used to describe the blood flow and pressure wave propagation phenomena in the arterial tree; geometrical multiscale models coupling the macrosvascular network (large arterials), mesovascular network (medium or small arterials) as well as microvascular network (arterioles or capillaries) are investigated for the

*Correspondence to: Modelling and Scientific Computing, CMCS, Mathematics Institute of Computational Science and Engineering, MATHICSE, Ecole Polytechnique Fédérale de Lausanne, EPFL, Station 8, CH-1015 Lausanne, Switzerland. Email: peng.chen@epfl.ch 
hope to simulate systemically the physiological blood flow in the entire human vascular network [30, 24, 28]. Moreover, models for tissue perfusion [18], mass transfer [50], bypass design [40], electromechanical activity of the heart [14] and so on have also been developed with specific objectives. Meanwhile, various efficient computational techniques [20, 35, 9, 8, 23, 17, 27] have also enhanced greatly for the cardiovascular modelling and simulation.

However, there are always discrepancies between measurements and the deterministic simulation results, since many uncertainties exist in cardiovascular system due to its complexity, diversity and variability [23]. For instance, the evolution of cerebral aneurysm can be influenced evidently by random external pressure or inflows [43]; the development of carotid atherosclerosis might be highly related to the inhomogeneous randomly distributed components of blood as well as the pattern of blood flow, whose geometry may be uncertain in a large degree due to the accumulation of fatty material [38]. Some of the uncertainties, namely epistemic uncertainties, can be reduced by more precise measurement or more advanced noise filtering techniques, while the other of them, namely aleatory uncertainties, are very difficult if not impossible to be accurately captured due to the inhomogeneous and multiscale properties of the cardiovascular system that undergoes an instantly and intrinsically variation owing to, for instance, the surrounding tissue pressure or external work force [23]. To identify and quantify these uncertainties, even if partially, and incorporate them into the well developed deterministic models will benefit not only for more accurate modelling and simulation of the cardiovascular system, but also for better understanding of the cardiovascular diseases. Therefore, development and analysis of efficient computational methods for uncertainty quantification becomes very important for mathematical modelling and numerical simulation of cardiovascular system.

In the modelling of the complex cardiovascular system, uncertainties are inevitably encountered from various sources and may play an important role in computational simulation. When it comes to mathematical modelling, these uncertainties may be classified in general in the following categories:

1. Computational geometries: Blood flow in the vascular system and the heart depends on the geometry of the blood lumen and blood vessel, which could be uncertain in a large extent, e.g., for what concerns, branch separation or bifurcation, high deflection bends, arterial wall thickness, lumen narrowed down by atherosclerosis or balloon-like bulge in the wall due to aneurysms. In fact, geometrical noise typically comes from the reconstruction of the geometry of the blood vessel with data from medical image devices, such as CT or MRI [38].

2. Mathematical models: There have been many mathematical models built in different geometrical scales and for different physical properties. Depending on the computational geometry, we may have multiscale models accounting for the detailed or simplified fluid velocity field and pressure with rigid or compliant walls. Based on the physical properties, we can characterize blood flow with Newtonian or non-Newtonian rheology, with or without viscoelastic or inertial properties, etc. [23]. Uncertainties thus arise from adoption of different mathematical models.

3. Physical parameters: When the mathematical model is established, the coefficients or physical parameters in the mathematical model is exposed to various uncertainties due to crude or unavailable measurements. The outcome of the blood flow simulation is undoubtedly determined at a certain extent by these physical parameters accounting for material properties of fluid and structure, such as the permeability, elasticity, compliance or Young's modulus of the arterial wall, the diffusivity of substance dissolved in the inhomogeneous blood solvent and so on [50].

4. Boundary conditions: Even for the same mathematical model and the same physical parameters, the confidence in the output of the computational simulation also depends on the uncertainties of boundary conditions prescribed on the boundary of the computational domain, including the inlet velocities or flow flux, outlet resistance or lumped parameter models, the interaction on the interface between the lumen and the arterial wall, etc [43].

5. External sources or forces: The transport of the main substance is carried by the blood, which also contains some other substances that make the chemical reaction between these different and contacting substances possible, resulting in increase or decrease of the substance 
of interest. Meanwhile, the flow rate of the blood is influenced by the surrounding tissue or uncertain distribution of external work force, which may also lead to the variation of the blood flow [23].

In order to have more precise characterization and interpretation of the cardiovascular system by mathematical modelling and numerical simulation, these uncertainties must be taken into account with different emphasis depending on the purpose. How to identify and propagate the influential uncertainties in the mathematical modelling on different levels is still at the beginning of investigation [43, 2, 49]. In fact, there is no mature techniques to characterize and represent these heterogeneous uncertainties arising from different sources, let alone systematic analysis of the influence of them to the cardiovascular system. Preliminary work has been carried out for parametric uncertainty quantification in a local region or part of the vascular network with only one or two types of parameters [43, 49]. The authors in [43] proposed an adaptive stochastic collocation framework for uncertainty quantification and propagation in cardiovascular simulations. They studied the radius of the abdominal aortic aneurysm, the radius and inflow velocity of the carotid artery bifurcation as well as flow split of the LPA/RPA as random variables following either Gaussian or uniform distribution to account for the uncertainty impact on blood flow modelled by three dimensional Navier-Stokes equations with rigid arterial wall in a small region. A main part of the vascular network with 37 arterial segments with independent and uniformly distributed parameters for each segment was described by a one dimensional stochastic model by the authors in [49]. In particular, they considered the sensitivity of pressure with respect to the uncertainty of material parameter (e.g. Young modulus) in different segments and apply generalized polynomial chaos combined with stochastic collocation method to compute the solution.

In this work, we concentrate on and highlight the following three aspects for uncertainty quantification in the human arterial tree: 1 , we first investigate several parametric uncertainties independently for time dependent sensitivity and stochastic convergence analysis; 2 , and then we study systemically many different sources of parametric uncertainties (around 10) listed above at the same time and examine their distinct importance to cardiovascular simulation via global sensitivity analysis; 3 , we also consider parameter dependent boundary conditions (resistance) in each distal boundary site and geometrical parameter (cross-section area) in each arterial segment obeying independent and identical probability distribution to unveil the most important region where the uncertainty is located for the quantity of interest. For this set of preliminary and explanatory analyses, we build a one dimensional stochastic fluid structure interaction model for the systemic arterial tree based on a one dimensional deterministic model validated by clinical measurements [39]. Although it couldn't be applied to study the local flow fields as in three dimensional modelling with detailed geometry, e.g. secondary flow, wall shear stress, vorticity of the velocity field, we are satisfied with this simplified one dimensional model for the fact that it is intrinsically a coupled hyperbolic system suitable to describe the blood flow and pressure wave propagation in the global vascular network. Moreover, we consider the vascular network with great completeness of the systemic circulation, incorporating the detailed description of the cerebral and coronary arteries, wall viscoelastic properties, wall friction and convection acceleration effect as well as realistic distal boundary conditions at the terminal sites characterized by three element windkessel model $[39,29]$. These advantages enable us in a large extent to carry out more realistic uncertainty quantification in the global vascular network with many parameters.

In section 2 we summarize the deterministic fluid structure interaction model of the human arterial tree with brief description of the coupling condition and the lumped parameter model for the terminal boundary as well as the basic numerical approximation scheme. Based on the deterministic model we formulate the stochastic model in the probability framework and introduce the stochastic collocation method to solve the stochastic system. Criteria for statistical and sensitivity analysis are defined according to the representation of stochastic collocation solution. In section 3, we study the statistics and sensitivity of the solution with respect to different uncertainties in three aspects: 1, stochastic regularity and nonlinearity of the solution as well as the convergence analysis of the collocation method in the case of one dimensional parametric uncertainty; 2, systemic time averaged and time dependent sensitivity analysis of all the uncertainties over one heart beat in the 
case of moderate (10) dimensional parametric uncertainty; 3 , quantification of the dependence of uncertainty location with independent random variables to account for uncertainty of resistance at each distal boundary and reference area in each arterial segment in the case of high (47 and 103) dimensional parametric uncertainty.

\section{MATHEMATICAL MODELLING OF THE HUMAN ARTERIAL TREE}

\subsection{Deterministic modelling: one dimensional fluid structure interaction}

We introduce the simplified one dimensional deterministic fluid structure interaction model to describe the blood flow in arteries and its interaction with the blood vessel displacement following [22, 36]. In the absence of bifurcation, each segment of the arteries can be considered as a cylindrical compliant and impermeable tube with cross section $S(t, x)$, being $t \in(0, T]$ the time and $x \in[0, L]$ the axial coordinate. The radius $r$ of the tube and the pressure $P$ of the blood flow are assumed to be functions of only $t$ and $x$. We also assume that the velocity $u$ of the blood flow is dominated in the axial coordinate and depends on $t, x$ and $r$ with the profile $s(r, \theta)=\theta^{-1}(\theta+2)\left(1-r^{\theta}\right)$, where we have $\theta=2$ for parabolic Poiseuille profile, $\theta=9$ for more physiological Womersley profile and $\theta \rightarrow \infty$ for a simple flat profile [23]. The state variables for the study of wave propagation phenomena, namely the cross-sectional area $A$, the volumetric flow rate $Q$ and the average pressure $P$, are defined by

$$
A(t, x)=\int_{S(t, x)} d S, \quad Q(t, x)=\int_{S(t, x)} u_{x} d S, \quad P(t, x)=\frac{1}{A} \int_{S(t, x)} P d S .
$$

By integrating the three dimensional Navier-Stokes equations on a generic section $S$ and replacing the velocity and pressure by the state variables defined in (1) (see [36, 23, 29] for details), we obtain the following simplified one dimensional equations governed by mass and momentum conservation law

$$
\left\{\begin{array}{c}
\frac{\partial A}{\partial t}+\frac{\partial Q}{\partial x}=0 \quad \text { in }(0, T] \times[0, L], \\
\frac{\partial Q}{\partial t}+\frac{\partial}{\partial x}\left(\alpha \frac{Q^{2}}{A}\right)+\frac{A}{\rho} \frac{\partial P}{\partial x}+k_{r} \frac{Q}{A}=0 \quad \text { in }(0, T] \times[0, L],
\end{array}\right.
$$

where the second term and the fourth term of the momentum equation account for the convective acceleration effect and the wall friction effect, respectively. $\alpha$ and $k_{r}$ are the Coriolis coefficient and friction coefficient defined as

$$
\alpha=\frac{1}{A} \int_{S(t, x)} s^{2} d S, \quad k_{r}=-\left.2 \pi \frac{\mu}{\rho} \frac{d s}{d r}\right|_{r=1},
$$

being $\mu$ the kinematic viscosity, $\rho$ the blood density and $r=1$ on the arterial wall.

In order to close the fluid system (2) consisting of two equations and three variables $A, Q$ and $P$, we need to provide an additional relation between the pressure and the wall deformation and thus the cross section area according to certain constitutive law of the arterial wall, for instance [36]

$$
P-P_{e x t}=\hat{\psi}(A)+\tilde{\psi}(A):=\beta\left(\sqrt{\frac{A}{A_{0}}}-1\right)+\gamma\left(\frac{1}{A \sqrt{A}} \frac{\partial A}{\partial t}\right),
$$

which incorporates not only the elastic effect in the first term but also the viscoelastic effect in the second term. The coefficients $\beta$ and $\gamma$ entail the physical parameters for the material property of the arterial wall in the following formula

$$
\beta=\sqrt{\frac{\pi}{A_{0}}} \frac{h E}{1-\nu^{2}}, \quad \gamma=\frac{T \tan \phi}{4 \sqrt{\pi}} \frac{h E}{1-\nu^{2}},
$$


where $h, E, \nu, A_{0}$ are the wall thickness, Young modulus, Poisson coefficient and reference sectional area under only external pressure $P_{\text {ext }}$; the viscoelastic parameters $T$ and $\phi$ are the wave characteristic time and angle determining the viscoelasticity effect. More complex wall models can also be employed by taking into account inertia, longitudinal elasticity, etc. [22]. Initial conditions for system (2) can be chosen in the reference state, being $A=A_{0}, Q=0$ and $P=P_{\text {ext }}$ or any other state with data extracted from either numerical simulations or clinical measurements [23].

For numerical approximation of system (2), we apply operator splitting techniques, decomposing the volumetric flow rate into two parts $Q=\hat{Q}+\tilde{Q}$ that account for the elastic effect and the viscoelastic effect respectively, and rewrite (2) for the elastic component in the conservation form [29]

$$
\frac{\partial \hat{U}}{\partial t}+\frac{\partial F(U)}{\partial x}+S(U)=0 \quad \text { in }(0, T] \times[0, L]
$$

where $U=[A, Q]^{T}$ and $\hat{U}=[A, \hat{Q}]^{T}$ are the total and conservative variables, $F=\left[Q, F_{2}\right]^{T}$ are the corresponding fluxes with

$$
F_{2}=\int_{A_{0}}^{A} \frac{A}{\rho} \frac{\partial \hat{\psi}}{\partial A} d A+\alpha \frac{Q^{2}}{A},
$$

and $S=\left[0, S_{2}\right]^{T}$ consists of the friction and the non-uniformity of the geometry and the material with

$$
S_{2}=k_{r} \frac{Q}{A}+\frac{A}{\rho}\left(\frac{\partial \hat{\psi}}{\partial A_{0}} \frac{\partial A_{0}}{\partial x}+\frac{\partial \hat{\psi}}{\partial \beta} \frac{\partial \beta}{\partial x}\right)-\frac{\partial}{\partial A_{0}} \int_{A_{0}}^{A} \frac{A}{\rho} \frac{\partial \hat{\psi}}{\partial A} d A \frac{\partial A_{0}}{\partial x}-\frac{\partial}{\partial \beta} \int_{A_{0}}^{A} \frac{A}{\rho} \frac{\partial \hat{\psi}}{\partial A} d A \frac{\partial \beta}{\partial x}
$$

As for the viscoelastic part, we obtain from system (2)

$$
\frac{1}{A} \frac{\partial \tilde{Q}}{\partial t}-\frac{\partial}{\partial x}\left(\frac{\gamma}{\rho A^{3 / 2}} \frac{\partial Q}{\partial x}\right)=0 \quad \text { in }(0, T] \times[0, L] .
$$

There have been several numerical discretization schemes applied to approximate (6) and (9), e.g. second order Taylor-Galerkin [22], discontinuous Galerkin [44], spectral/hp element [49]. We choose the second order Taylor-Galerkin finite element approximation for simplicity, which is more convenient to deal with the operator splitting techniques [29]. It can be shown [36] that (6) is a first order non-linear hyperbolic system so that some compatibility condition is needed to enable the computation of state variables at the first and last nodes. Moreover, the system (6) and (9) are closed by imposing valid boundary conditions, such as physiological flow rate, terminal absorbing conditions or lumped parameter models, for instance, the three element windkessels consisting of two resistors and one capacitor described by the ordinary differential equation

$$
P-P_{v}+C R_{2} \frac{d P}{d t}=\left(R_{1}+R_{2}\right) Q+C R_{1} R_{2} \frac{d Q}{d t} \quad \text { in }(0, T],
$$

where $P_{v}$ is the prescribed venous pressure, $C$ is the capacitance and $R_{1}, R_{2}$ are the resistances with the common relation $R_{1}=4 R / 5, R_{2}=R / 5$ where $R=R_{1}+R_{2}$ is the total resistance [39].

The one dimensional fluid structure interaction model (2) and (4) together with proper initial and boundary conditions is among the most popular way to describe the blood flow and its interaction with the arterial wall in each arterial segment locally [39]. In order to construct all the segments structurally and functionally to form the human arterial tree, suitable coupling conditions are needed at the bifurcations. It is demonstrated in [22] that a domain decomposition approach by keeping the mass conservation and total pressure continuity are satisfactory for characterizing blood velocity and pressure wave propagation without evident dissipation of energy in the arterial branching. More explicitly, supposing there are $N_{p}$ proximal segments and $N_{d}$ distal segments at a certain joint, we impose the following equations for the proximal and distal state variables $\left(Q^{p}, P^{p}\right)$ and $\left(Q^{d}, P^{d}\right)$

$$
\sum_{n=1}^{N_{p}} Q_{n}^{p}=\sum_{m=1}^{N_{d}} Q_{m}^{d}, \quad \text { and } \quad P_{n}^{p}=P_{m}^{d}, \forall n=1, \ldots, N_{p}, m=1, \ldots, N_{d} .
$$


This approach can be realized numerically by nonlinear Richardson strategy, using Newton or inexact Newton method combined with Broyden algorithm for updating Jacobian matrix, see [29] for details.

\subsection{Stochastic modelling: quantifying uncertainties}

As introduced in section 1, there are many different kinds of uncertainties arising from diverse sources of the human arterial tree with distinct features. Among several possible approaches to describe these uncertainties, e.g., evident theory, fuzzy set theory, probability theory, the latter ones provide a general framework to characterize various uncertainties more precisely in a quantitative way $[7,5]$. Roughly speaking, we employ the concept of random variable in probability theory to randomize a deterministic variable or parameter, e.g. the reference area $A_{0}(x) \in \mathbb{R}, x \in[0, L]$, to a real-valued random variable depending also on some outcome $\omega$ of a set of probability events $\Omega$, i.e. $A_{0}(x, \omega) \in \mathbb{R}, x \in[0, L], \omega \in \Omega$. Once the deterministic variable is replaced by a random variable, the model consisting of (6), (9) and (10) becomes a stochastic model. In order to solve the stochastic model, we first take a series of stochastic realization $A_{0}\left(x, \omega_{n}\right), n=1,2, \ldots, N$ according to certain prescribed probability distribution, then solve a deterministic model for each $A_{0}\left(x, \omega_{n}\right), n=1,2, \ldots, N$. For the sake of evaluating statistics of the stochastic solution, e.g. expectation or mean value where we need to compute an integral of the solution with respect to a probability distribution, we use properly selected quadrature formulas based on a set of collocation points and weights, which is called stochastic collocation method [4]. When the number of random variables becomes large, for instance $K$ random variables, we need $N^{K}$ collocation points for the full tensor product stochastic collocation method, yielding $N^{K}$ deterministic models to solve, which is computationally prohibitive especially when the solution of a single deterministic model is expensive. In this case, we employ sparse grid stochastic collocation method [32], i.e. select some representative collocation points to form a sparse grid to alleviate considerably the computational effort. In general, this method features more accurate and efficient evaluation of the statistics of stochastic solution. Let us introduce the procedure more rigorously.

Given a complete probability space $(\Omega, \mathcal{F}, \mathcal{P})$, which consists of the set of outcomes $\Omega$, the $\sigma$ algebra of events $\mathcal{F}$ and a probability measure $\mathcal{P}: \mathcal{F} \rightarrow[0,1]$, we can define a random variable $Y: \Omega \rightarrow \mathbb{R}$ such that for every Borel set $B \subset \mathbb{R}$ we have $Y^{-1}(B)=\{\omega \in \Omega: Y(\omega) \in B\} \in \mathcal{F}$. Provided that the random variable depends also on temporal or spatial coordinate, we have stochastic process or random fields [7]. The stochastic system of the one dimensional fluid structure interaction model corresponding to the deterministic system (6), (9) and (10) becomes: find the stochastic state processes $(A, Q, P):(0, T] \times[0, L] \times \Omega \rightarrow \mathbb{R}^{3}$, such that $\mathcal{P}$-almost surely the following equations hold with proper initial boundary conditions:

$$
\left\{\begin{array}{l}
\frac{\partial \hat{U}}{\partial t}(t, x, \omega)+\frac{\partial F(U)}{\partial x}(t, x, \omega)+S(U)(t, x, \omega)=0 \quad \text { in }(0, T] \times[0, L] \times \Omega, \\
\frac{1}{A} \frac{\partial \tilde{Q}}{\partial t}(t, x, \omega)-\frac{\partial}{\partial x}\left(\frac{\gamma}{\rho A^{3 / 2}} \frac{\partial Q}{\partial x}\right)(t, x, \omega)=0 \quad \text { in }(0, T] \times[0, L] \times \Omega, \\
P(t, \omega)-P_{v}+C R_{2} \frac{d P}{d t}(t, \omega)=\left(R_{1}+R_{2}\right) Q(t, \omega)+C R_{1} R_{2} \frac{d Q}{d t}(t, \omega) \quad \text { in }(0, T] \times \Omega .
\end{array}\right.
$$

For each realization $\omega \in \Omega$, we assume that the stochastic system (12) shares the same mathematical properties as its deterministic counterpart, in particular, being still a hyperbolic system. The stochasticity of the state variables is propagated from the random inputs accounting for the uncertainties of involved parameters in the system, including the geometrical parameters, physical parameters as well as parameters arising from boundary conditions and external pressure. These parameters can be either represented by random variables or stochastic processes following certain probability distributions. In order to calibrate the probability distributions from various data sources such as literatures, measurements or experts' opinions, we may employ statistical inference techniques, for instance, linear and nonlinear regression, maximum likelihood estimation, maximum entropy, etc. [19, 46]. 
In the absence of sufficient data for calibration, we assume a general log-normal distribution for the parametric uncertainties with two considerations: 1, the deterministic value available in the literature, e.g. [39], is the most likely value with high probability (close to the expectation) while the value far from this center has low probability (with small deviation), for which normal or lognormal distribution qualifies by the maximum entropy theory [19]; 2 , the parametric uncertainties should not change the sign of the parameter value, i.e. the probability to change the sign should be (or be close to) zero, for which the log-normal distribution is more appropriate since its image is $\mathbb{R}_{+}$. Without loss of generality, we assume the random parameter perturbed by a log-normal distributed random variable as

$$
\eta(t, x, \omega)=e^{\mu_{e}+\sigma_{v} Y(\omega)} \eta(t, x),
$$

where $\eta(t, x)$ is the deterministic parameter under consideration, which might depend on time and space, and $\eta(t, x, \omega)$ is the randomized parameter; $Y$ is a random variable obeying standard normal distribution, which is transformed by two prescribed parameters $\mu_{e}, \sigma_{v}$ to a log-normal random variable as $X(\omega)=\exp \left(\mu_{e}+\sigma_{v} Y(\omega)\right)$ with expectation $\mathbb{E}[X]=\exp \left(\mu_{e}+\sigma_{v}^{2} / 2\right)$, variance $\mathbb{V}[X]=\left(\exp \left(\sigma_{v}^{2}\right)-1\right) \exp \left(2 \mu_{e}+\sigma_{v}^{2}\right)$, standard deviation $\mathbb{S}[X]=\sqrt{\mathbb{V}[X]}$ and the mode $\exp \left(\mu_{e}-\right.$ $\sigma_{v}^{2}$ ) (where the density function is maximized). Figure 1 displays the probability density functions of the random variable $Y$ following standard normal distribution (left) and the transformed random variable $X$ following log-normal distribution with different values of the parameter $\mu_{e}, \sigma_{v}$ (right), which represent different degrees of uncertainty characterized by signal-to-noise ratio $(\mathrm{SNR}=$ $\mathbb{E}[X] / \mathbb{S}[X][11])$. We choose the mode as 1 so that $\mu_{e}=\sigma_{v}^{2}$ to guarantee that the deterministic value of parameter $\eta$ has the highest probability. For the choice $\mu_{e}=0.01, \sigma_{v}=0.1$ we have SNR $\approx 9.975$, or the noise-to-signal ratio $1 / \mathrm{SNR} \approx 10 \%$, for $\mu_{e}=0.0625, \sigma_{v}=0.25$, we have $1 / \mathrm{SNR} \approx$ $25.4 \%$ and $\mu_{e}=0.25, \sigma_{v}=0.5$, we have a relatively large noise-to-signal ratio $1 / \mathrm{SNR} \approx 53.3 \%$.
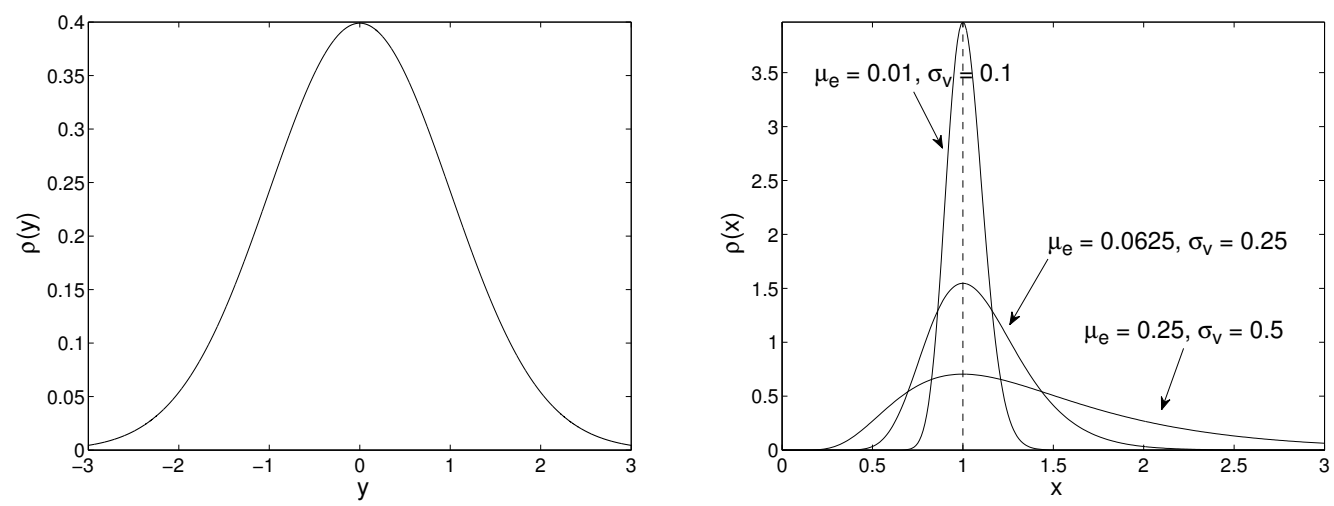

Figure 1. Left, standard normal density function; right, several log-normal density functions

Supposing that the random inputs can be represented by $K$ random variables $Y=\left(Y_{1}, \ldots, Y_{K}\right)$ with joint probability density functions $\rho(Y)=\Pi_{k=1}^{K} \rho_{k}\left(Y_{k}\right)$ calibrated from available data, we have that the stochastic state processes depend on the uncertainties only through $Y$ in the image $\Gamma:=Y(\Omega)$, i.e. $(A, Q, P):(0, T] \times[0, L] \times \Gamma \rightarrow \mathbb{R}^{3}$. In another word, we can view the stochastic system (12) as a parametrized system. Therefore the random realization $\omega \in \Omega$ in (12) can be replaced by the parameter $y \equiv Y(\omega) \in \Gamma$ and to solve the stochastic system (12) is equivalent to solve a deterministic system at each parameter $y \in \Gamma$. The transformation from stochastic system to parametric system is the starting point for the application of fast stochastic computational methods that have been developed recently, including stochastic collocation method [48, 4], stochastic Galerkin method [7], reduced basis method [10], spectral decomposition method [33], low rank tensor Krylov subspace method [26] and so on. Provided that the solution of the parametric system is smooth enough with respect to the parameter $y$, these methods have been proved to achieve exponential or sub-exponential convergence rate, much faster than the algebraic convergence rate 
$N^{-1 / 2}$ of Monte-Carlo method [21]. Moreover, stochastic collocation method, similar to MonteCarlo method, turns out more applicable for nonlinear and complex system due to its non-intrusive feature that enables us to use the deterministic solver directly and repeatedly without mathematical reformulation $[6,15]$.

Given the collocation points in $\Gamma \subset \mathbb{R}$, e.g., $-\infty<y^{0}<y^{1}<y^{2}<\cdots<y^{N}<\infty$ as well as the corresponding functions $f\left(y^{n}\right), 0 \leq n \leq N$ (state variables $(A, Q, P)$ in our context), we define the univariate $N$ th order Lagrangian interpolation

$$
\mathcal{U}_{N} f(y)=\sum_{n=0}^{N} f\left(y^{n}\right) l^{n}(y), \quad \text { where } l^{n}(y)=\prod_{m \neq n} \frac{y-y^{m}}{y^{n}-y^{m}} \quad 0 \leq n \leq N .
$$

Rewrite the univariate interpolation formula (14) with the index $k$ for the $k$ th dimension as

$$
\mathcal{U}_{N_{k}} f\left(y_{k}\right)=\sum_{y_{k}^{n_{k}} \in \Theta^{k}} f\left(y_{k}^{n_{k}}\right) l_{k}^{n_{k}}\left(y_{k}\right), \text { where } \Theta^{k}=\left\{y_{k}^{n_{k}} \in \Gamma_{k}, n_{k}=0, \ldots, N_{k}\right\} \text { for some } N_{k} \geq 1
$$

then the multivariate interpolation is given as the tensor product of the univariate interpolation

$$
\left(\mathcal{U}_{N_{1}} \otimes \cdots \otimes \mathcal{U}_{N_{K}}\right) f(y)=\sum_{y_{1}^{n_{1}} \in \Theta^{1}} \cdots \sum_{y_{K}^{n_{K} \in \Theta^{K}}} f\left(y_{1}^{n_{1}}, \ldots, y_{K}^{n_{K}}\right)\left(l_{1}^{n_{1}}\left(y_{1}\right) \otimes \cdots \otimes l_{K}^{n_{K}}\left(y_{K}\right)\right) .
$$

In order to alleviate the "curse of dimensionality" in the interpolation on the full tensor product grid for high dimensional problems, we employ the Smolyak sparse grid interpolation that considerably reduces the number of the total collocation nodes by removing most of the cross-dimensional collocation nodes in an optimizing way while keeping high order interpolation in each dimension [45]. It achieves fast convergence rate without much sacrifice of accuracy compared to the same level of the full tensor product interpolation, in particular for analytic problem, and is proven to be one of the most efficient and widely used stochastic collocation methods [48, 32]. The general Smolyak formula reads

$$
\mathcal{S}_{q} f(y)=\sum_{q-K+1 \leq|i| \leq q}(-1)^{q-|i|}\left(\begin{array}{c}
K-1 \\
q-|i|
\end{array}\right)\left(\mathcal{U}^{i_{1}} \otimes \cdots \otimes \mathcal{U}^{i_{K}}\right) f(y),
$$

where $|i|=i_{1}+\cdots+i_{K}$ with the multivariate index $i=\left(i_{1}, \ldots, i_{K}\right)$ defined via the two possible sets

$$
X_{s}(q, K):=\left\{i \in N_{+}^{K}, \forall i_{k} \geq 1: \sum_{k=1}^{K} i_{k} \leq q\right\} \text { or } X_{p}(q, K):=\left\{i \in N_{+}^{K}, \forall i_{k} \geq 1: \prod_{k=1}^{K} i_{k} \leq q\right\}
$$

and the set of collocation nodes of the sparse grid is thus collected as

$$
H(q, K)=\bigcup_{q-K+1 \leq|i| \leq q}\left(\Theta^{i_{1}} \times \cdots \times \Theta^{i_{K}}\right)
$$

where $\# \Theta^{i_{k}}=1$ if $i_{k}=1$, and $\# \Theta^{i_{k}}=2^{i_{k}-1}+1$ when $i_{k}>1$ in a nested structure. Note that we denote $\mathcal{U}^{i_{k}} \equiv \mathcal{U}_{N_{k}}$ defined in (15) for $N_{k}=2^{i_{k}-1}$. We define $q-K$ as the level of interpolation. Figure 2 depicts the full tensor product grid, sparse grid with index sets $X_{s}(q, K)$ and $X_{p}(q, K)$ with collocations nodes defined as Gauss-Hermite quadrature abscissas [13] for two independent random variables obeying standard normal distribution, from which we can observe a large reduction of the total number of collocation nodes, especially for the sparse grid with index set $X_{p}(q, K)$. More advanced techniques, such as anisotropic sparse grid [31], sparse grid constructed via hierarchical surplus [25] and reduced basis [15], have been developed by taking advantage of stochastic regularity and a posteriori error estimate for further reduction of the computational cost. 

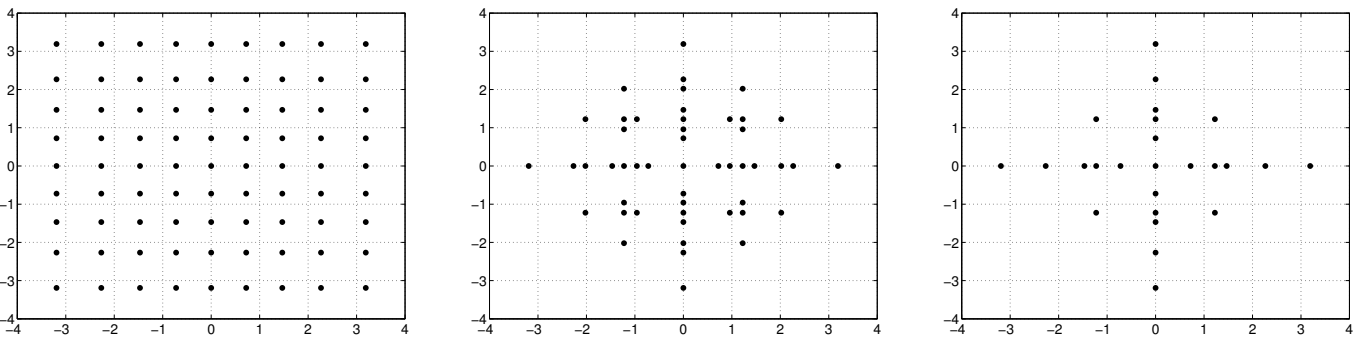

Figure 2. Two dimensional ( $K=2$ ) full tensor product grid (left, 81 nodes) and sparse grid with index set $X_{s}(q, K)$ (middle, 49 nodes) and index set $X_{p}(q, K)$ (right, 25 nodes), all the collocation nodes are chosen as Gauss-Hermite quadrature abscissas with the level of interpolation $q-K=3$

By repeatedly solving a deterministic system at each collocation node (19), we can construct an explicit formula for the stochastic state variables at any parameter $y \in \Gamma$ via the sparse grid interpolation (17). In practice, we are more interested in the evaluation of the statistics of the state variables, such as expectation or variance, which can be computed straightforwardly as

$\mathbb{E}[f] \approx \mathbb{E}\left[\mathcal{S}_{q} f\right]=\int_{\Gamma} \mathcal{S}_{q} f(y) \rho(y) d y=\sum_{q-K+1 \leq|i| \leq q}(-1)^{q-|i|}\left(\begin{array}{c}K-1 \\ q-|i|\end{array}\right) \mathbb{E}\left[\left(\mathcal{U}^{i_{1}} \otimes \cdots \otimes \mathcal{U}^{i_{K}}\right) f\right]$

where the tensor product expectation can be evaluated by the following quadrature formula

$$
\mathbb{E}\left[\left(\mathcal{U}_{N_{1}} \otimes \cdots \otimes \mathcal{U}_{N_{K}}\right) f\right]=\sum_{y_{1}^{n_{1}} \in \Theta^{1}} \cdots \sum_{y_{K}^{n_{K}} \in \Theta^{K}} f\left(y_{1}^{n_{1}}, \ldots, y_{K}^{n_{K}}\right)\left(w_{1}^{n_{1}} \times \cdots \times w_{K}^{n_{K}}\right),
$$

being $\left(y_{k}^{n_{k}}, w_{k}^{n_{k}}\right), 1 \leq k \leq K$ the quadrature abscissas and weights according to the joint probability distribution function and $\mathcal{U}_{N_{k}} \equiv \mathcal{U}^{i_{k}}, 1 \leq k \leq K$. The evaluation of variance is computed by

$$
\mathbb{V}[f]=\mathbb{E}\left[(f-\mathbb{E}[f])^{2}\right] \approx \mathbb{E}\left[\mathcal{S}_{q} f^{2}\right]-\left(\mathbb{E}\left[\mathcal{S}_{q} f\right]\right)^{2} .
$$

In order to improve the accuracy of the numerical integral in (20) and the numerical interpolation in (17), it is favourable to select the collocation points as the quadrature abscissas. Available quadrature rules include Clenshaw-Curtis quadrature (with Chebshev Gauss Lobatto nodes), Gaussian quadrature based on various orthogonal polynomials and so on [37, 32].

Another interest is to study the sensitivity of the state variables with respect to different parameters, or in another word, how the solution depends on each parameter at some realization $y \in \Gamma$, namely local or pointwise sensitivity analysis [41], as well as how much weight that the uncertainty arising from each parameter contributes to the total variation of the solution in the name of global sensitivity analysis [42, 12]. In the uncertainty quantification of the human arterial tree, we are more interested in how different parameters affect the blood flow and pressure wave propagation systemically, therefore, the global sensitivity analysis. Following [12], we define the variance based global sensitivity index - main effect of the $k$ th parameter as

$$
\mathbb{G}_{k}[f]=\frac{\mathbb{V}\left[\mathbb{E}\left[f \mid y_{k}\right]\right]}{\mathbb{V}[f]}, \quad k=1, \ldots, K,
$$

where $\mathbb{V}\left[\mathbb{E}\left[f \mid y_{k}\right]\right]$ is the variance of the expectation of the variable $f$ conditioned on the $k$ th parameter $y_{k}$, accounting for the contribution to the total variance of the solution by this parameter. More explicitly, it can be evaluated approximately via the sparse grid interpolation by

$$
\mathbb{V}\left[\mathbb{E}\left[f \mid y_{k}\right]\right] \approx \int_{\Gamma_{k}}\left(\int_{\Gamma_{k}^{*}} \mathcal{S}_{q} f(y) \rho\left(y_{k}^{*}\right) d y_{k}^{*}\right)^{2} \rho\left(y_{k}\right) d y_{k}-\left(\int_{\Gamma} \mathcal{S}_{q} f(y) \rho(y) d y\right)^{2},
$$


where $y_{k}$ is the $k$ th element of $y$ in one dimensional parameter space $\Gamma_{k}$ and $y_{k}^{*}$ is the adjoint counterpart or all the other elements of $y$, a $K-1$ dimensional parameter living in the parameter space $\Gamma_{k}^{*}$. The advantage of (23) attributes to its ability to measure the relative importance of different parameters and thus provide a guide for the effort (spent on collecting data, selecting statistical inference techniques, etc.) to quantify the uncertainty arising from each of them.

\section{SOME NUMERICAL RESULTS AND ANALYSIS}

\subsection{Set up of simulation}

We take the one dimensional human arterial tree with schematic representation from [39], see Figure 3 for details. It represents the main systemic arterial tree with great completeness, including the aortic arch and the coronary network, the principal abdominal aorta branches as well as the cerebral arterial tree. There are 103 segments in the arterial tree, indexed from 1 to 103 , thus 103 one dimensional fluid structure interaction models (2) coupled together at the junctions by (11). Each of the 47 distal boundaries are described by one lumped parameter model (10). At the proximal boundary of ascending aorta 1 (1), a physiological flow rate over one heart beat of 0.8 second is imposed as the boundary condition. The value of geometrical parameters including arterial segment length and lumen diameter as well as the terminal resistance and compliance are set according to the data presented in [39]. The parameter $\theta$ for the velocity profile is set to 9 , leading to a more physiological Womersley flow. Poisson coefficient $\nu=0.5$ represents an incompressible arterial wall. Wall thickness $h$, Young modulus $E$, characteristic time $T$ and characteristic angle $\phi$ are chosen the same as in [29].
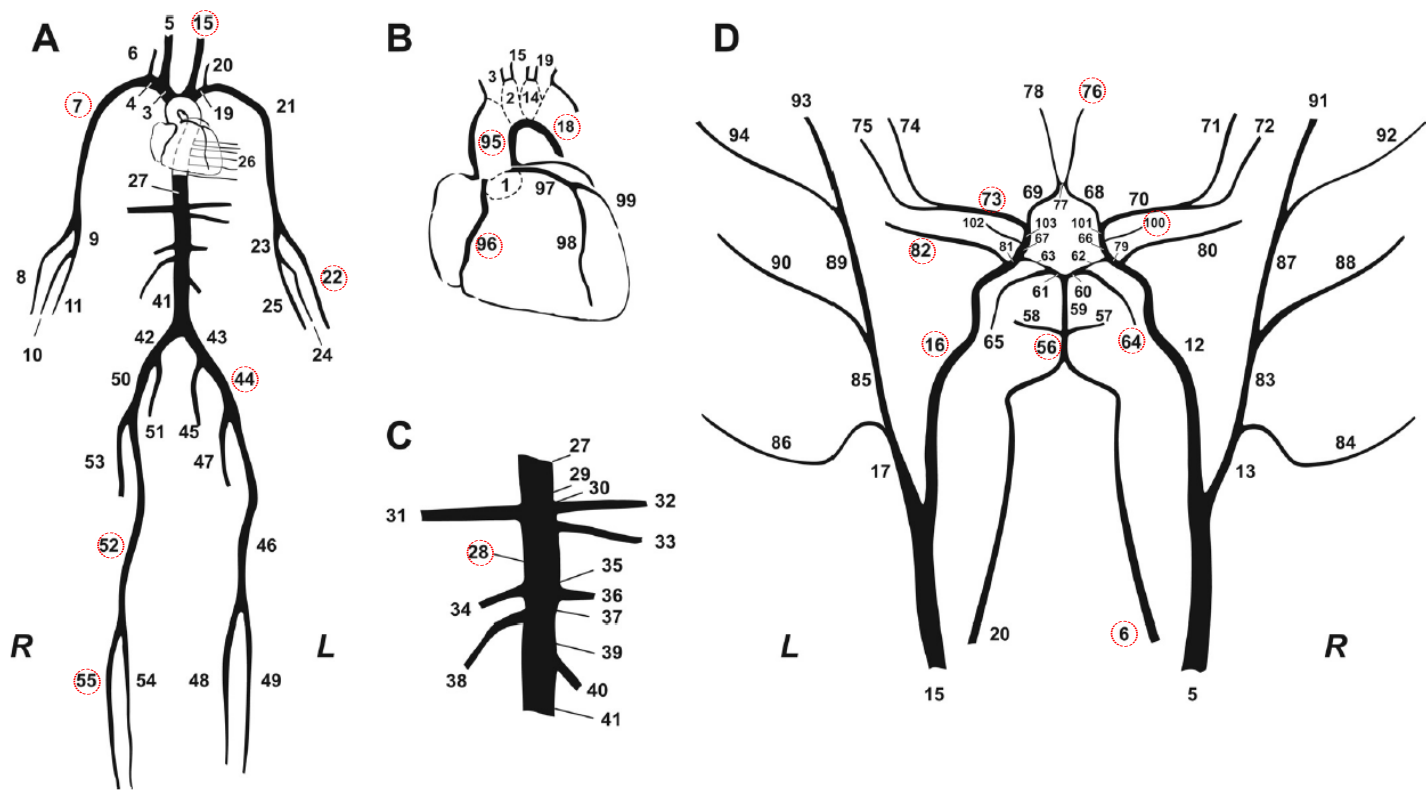

Figure 3. Schematic representation of the human arterial tree, taken from [39]. A: main systemic arterial tree. B: detail of the aortic arch and the coronary network. C: detail of the principal abdominal aorta branches. D: blown-up schematic of the detailed cerebral arterial tree, connected via the carotids (segments 5 and 15) and the vertebrals (segments 6 and 20) to the main arterial tree. R: right; L: left.

For physiological considerations, we are mainly interested in the blood flow rate and pressure at the following 18 representative locations ( 8 in the cerebral arterial tree and 10 in the main arterial tree) marked with circle in Figure 3: right coronary RCA (96), ascending aorta 2 (95), left common carotid (15), left radial (22), abdominal aorta A (28), left external iliac (44), right anterior tibial (55), 
right femoral (52), thoracic aorta A (18), right subclavian B, axillary, brachial (7), middle cerebral M1 (73), right ant. cerebral A2 (76), right ant. choroidal (100), right post. cerebral 2 (64), basilar artery 2 (56), right vertebral (6), left internal carotid (16), left ophthalmic (82).

We implement the solver for the coupled stochastic system (12) based on the deterministic solver implemented in LifeV [1], a parallel library written in $\mathrm{C}++$, for the one dimensional fluid structure interaction model of the human arterial tree. The spatial and temporal discretization are specified as 2 mesh elements for 1 centimeter and 2 milliseconds per time step, respectively. Piecewise linear polynomial functions are used as the finite element bases. The output of blood flow rate and pressure is taken in the time interval of the sixth heart beat (4.0 - 4.8 seconds), when the simulation reaches a relatively stable periodic state. Although the discretization has been rather crude in order to reduce the computational time, it is fine enough to capture the right wave propagation phenomena accurately compared to a finer discretization presented in [29]. It takes around 25 minutes to run the simulation for six heart beats by 16 processors (Intel Xeon Nehalem $2.66 \mathrm{GHz}$ ). Thanks to the nonintrusive property of the stochastic collocation method, we can run the stochastic simulation at each random realization or collocation node in a complete parallel structure. For instance, it takes around 50 hours to run the stochastic simulations with the second level of interpolation for 10 random variables or the first level of interpolation for 103 random variables by $10 \times 16$ processors.

\subsection{One dimensional parametric uncertainty - preliminary analysis}

The boundary conditions - prescribed physiological flow rate $Q(t)$ and terminal resistance at different locations $R$ (location), the geometrical parameter - reference area of the arterial wall $A_{0}(x)$, the physical parameter - Young modulus $E(x)$ and many other parameters, are different among people of different ages, sizes, genders and other factors. Even for the same person, these parameters may vary according to the work effort, healthy state, etc. In this section we study independently the uncertainty effect of these parameters to the blood flow and pressure wave propagation with different degrees of uncertainties. We first define the a posteriori error of the statistics (expectation and standard deviation) approximated by the stochastic collocation method in level $l=q-K$ (17) as

$$
\operatorname{error}\left(\mathbb{E}_{l}[f]\right)=\frac{\left\|\mathbb{E}\left[\mathcal{S}_{q+1} f\right]-\mathbb{E}\left[\mathcal{S}_{q} f\right]\right\|}{\left\|\mathbb{E}\left[\mathcal{S}_{q+1} f\right]\right\|}, \quad \operatorname{error}\left(\mathbb{S}_{l}[f]\right)=\frac{\left\|\mathbb{S}\left[\mathcal{S}_{q+1} f\right]-\mathbb{S}\left[\mathcal{S}_{q} f\right]\right\|}{\left\|\mathbb{S}\left[\mathcal{S}_{q+1} f\right]\right\|}
$$

where the norm $\|v\|$ is space and time averaged value of the quantity $v$.
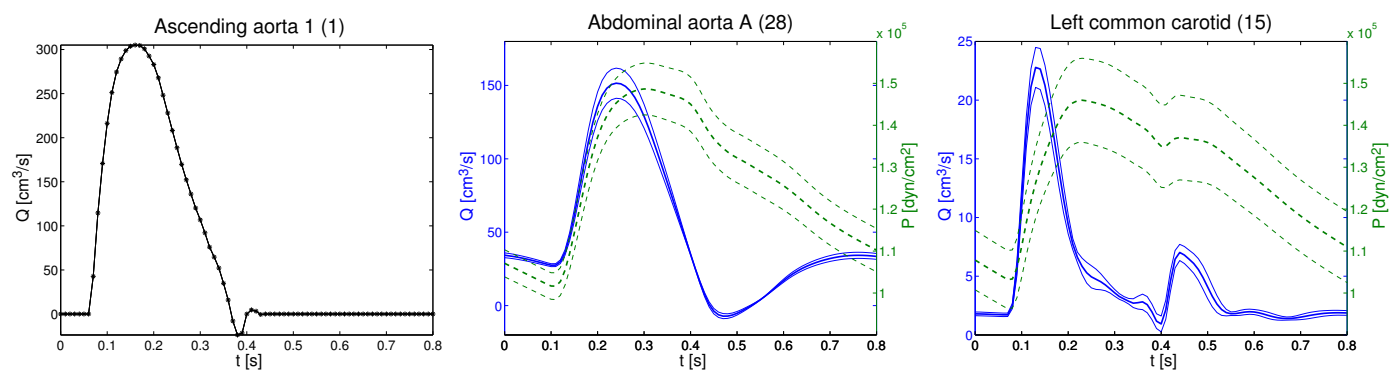

Figure 4. Imposed physiological flow rate for one heart beat (left), expectation with deformation by standard deviation of flow rate and pressure at the locations 28 and 15 during the sixth heart beat

The prescribed physiological flow rate for one heart beat is displayed on the left of Figure 4, which is randomized by a log-normal distributed random variable $X(\omega)=\exp \left(\mu_{e}+\sigma_{v} Y(\omega)\right)$ (13) with $\mu_{e}=0.01, \sigma_{v}=0.1$ and $Y$ follows standard normal distribution, see Figure 1 . The statistics are computed via (20) and (22). The expectation $\mathbb{E}[\cdot]$ and expectation with deformation by standard deviation $\mathbb{E}[\cdot]-\mathbb{S}[\cdot]$ and $\mathbb{E}[\cdot]+\mathbb{S}[\cdot]$ of the flow rate and the pressure at the location of abdominal aorta A (28) and left common carotid (15) are shown in Figure 4, from which we can observe that both quantities display some uncertainty effect due to the prescribed random flow rate and it has a relatively larger impact on the pressure (change in mean value) than on the flow rate (change in 

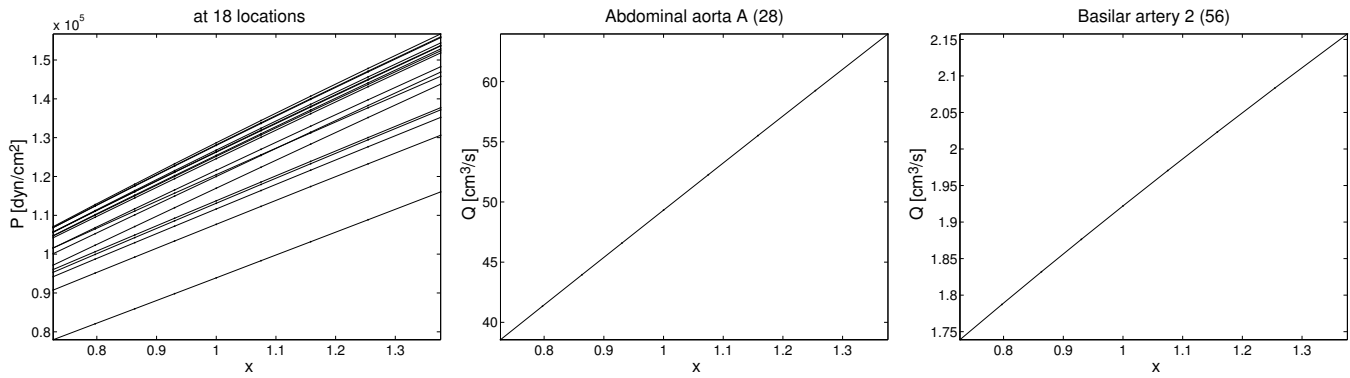

Figure 5. The dependence of pressure (left) and flow rate with respect to the random variable $X$
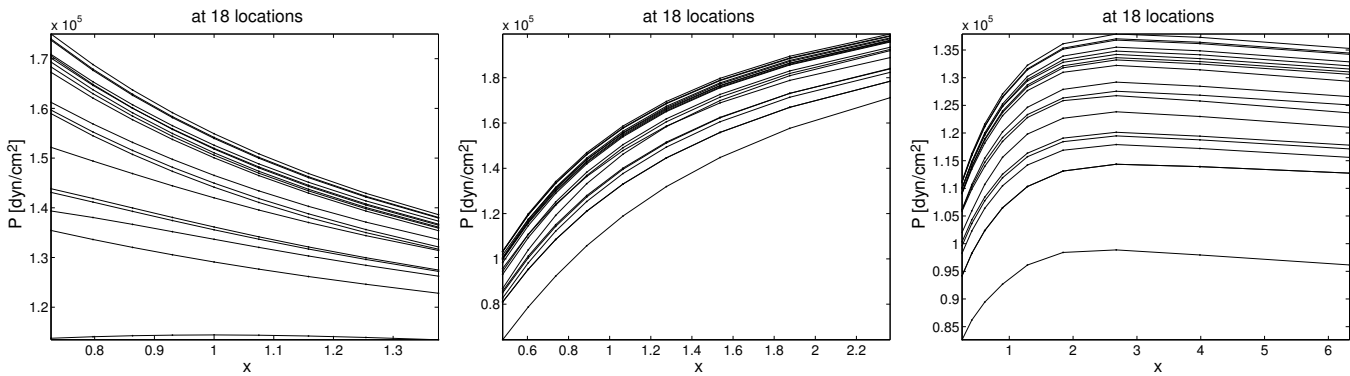

Figure 6. The dependence of pressure with respect to different random variables $X$ accounting for the uncertainties of the parameters area $A_{0}$ (left), resistance $R$ (middle) and Young modulus $E$ (right)
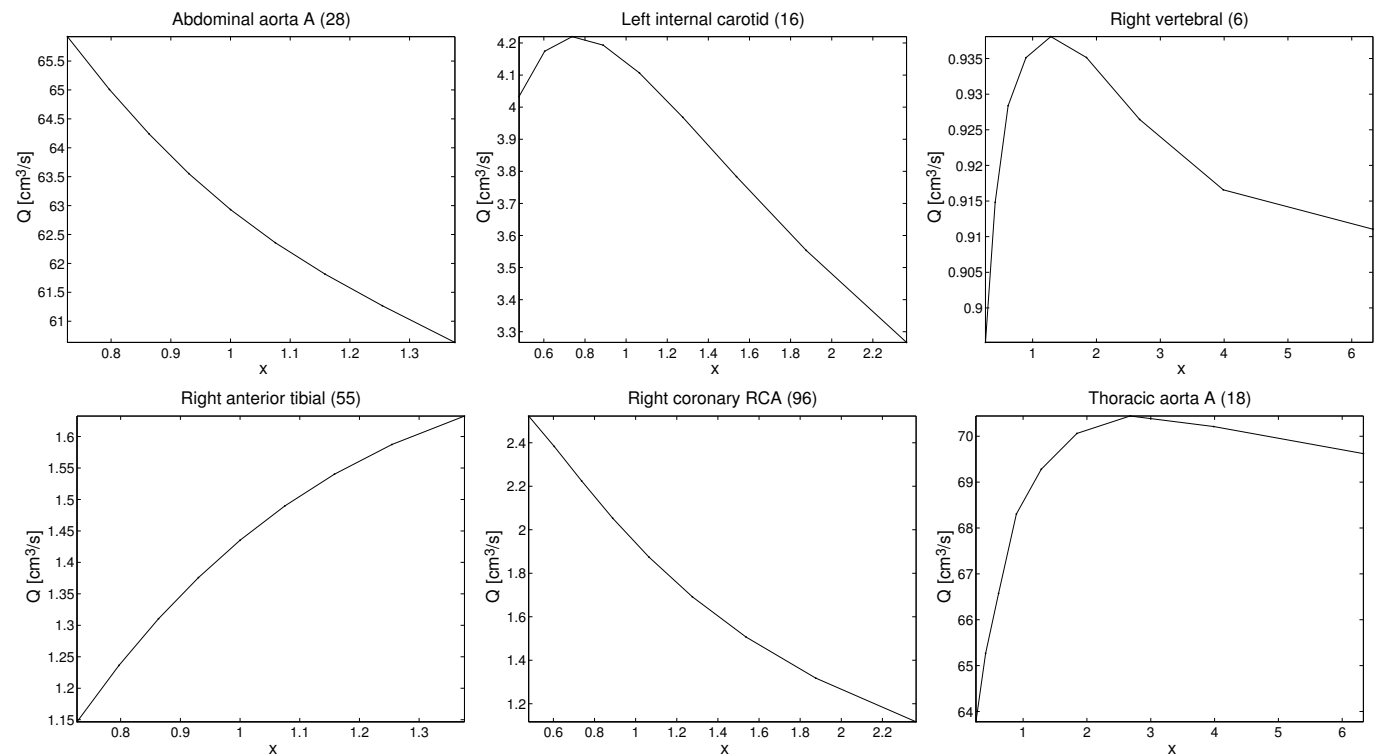

Figure 7. The dependence of flow rate with respect to different random variables $X$ accounting for the uncertainties of the parameters area $A_{0}$ (left), resistance $R$ (middle) and Young modulus $E$ (right)

wave shape) at both locations. We remark that uncertainties arising from the shape of the prescribed inflow rate or the frequency of heart beat can also lead to variation of both the mean value and the wave shape of the blood flow rate and the pressure due to different wave propagation and refection features. However, this kind of uncertainty is beyond our consideration in the present work. 
Figure 5 depicts the dependence of pressure at all the locations and flow rate at two representative locations (magnitude is different at different locations), from which we can tell that the pressure and flow rate increase linearly with respect to the random variable $X$, which implies that in order to obtain accurate first (expectation) and second (variance) moments of the output, we only need a small level of interpolation by the stochastic collocation method, which is verified from the relative error in Table I, i.e. the error of the second level of interpolation is just slightly smaller than that of the first level of interpolation. *

\begin{tabular}{|c|cc|cc|cccc|ccc|}
\hline parameter & \multicolumn{2}{|c|}{ flow rate $Q$} & \multicolumn{2}{|c|}{ area $A_{0}$} & \multicolumn{3}{c|}{ resistance $R$} & \multicolumn{3}{c|}{ Young modulus $E$} \\
\hline level $l$ & 1 & 2 & 1 & 2 & 1 & 2 & 3 & 1 & 2 & 3 \\
\hline error $\left(\mathbb{E}_{l}[Q]\right)$ & 0.012 & 0.008 & 0.078 & 0.048 & 0.013 & 0.011 & 0.007 & 11.687 & 3.186 & 0.422 \\
error $\left(\mathbb{S}_{l}[Q]\right)$ & 0.317 & 0.236 & 3.218 & 2.356 & 1.469 & 0.226 & 0.165 & 92.540 & 39.088 & 8.959 \\
error $\left(\mathbb{E}_{l}[P]\right)$ & 0.003 & 0.002 & 0.011 & 0.007 & 0.003 & 0.003 & 0.002 & 1.677 & 0.492 & 0.075 \\
error $\left(\mathbb{S}_{l}[P]\right)$ & 0.068 & 0.051 & 0.656 & 0.438 & 0.082 & 0.025 & 0.020 & 42.766 & 15.812 & 2.565 \\
\hline
\end{tabular}

Table I. A posteriori error of the statistics (25) for different parameters in different levels $\left(\times 10^{-3}\right)$

As for the geometrical parameter $A_{0}$, terminal resistance $R$ and Young modulus $E$, we use $\left(\mu_{e}=0.01, \sigma_{v}=0.1,1 / \mathrm{SNR} \approx 10 \%\right),\left(\mu_{e}=0.0625, \sigma_{v}=0.25,1 / \mathrm{SNR} \approx 25.4 \%\right),\left(\mu_{e}=\right.$ $\left.0.25, \sigma_{v}=0.5,1 / \mathrm{SNR} \approx 53.3 \%\right)$, respectively, to distinguish the different degrees of uncertainties in (13). The dependence of pressure at all the locations and flow rate at some representative locations with respect to different uncertainties are shown in Figure 6 and 7. It is quite evident that all these quantities display nonlinear dependence on the uncertainties with high stochastic regularity. In particular, the pressure decreases as the area of the lumen increases and becomes more flat when the area becomes large enough, which is in accordance with physiological flow. On the other hand, the pressure increases as both the resistance and Young modulus increase, and start to slightly decrease when the Young modulus becomes large enough. Different from the pressure, the flow rate depends on the uncertainty in a distinct manner in different locations of the arterial tree for all the parameters as can be observed in Figure 7. This is verified from the value of the error $\left(\times 10^{-3}\right)$ in Table I, where the error of expectation and standard deviation of the flow rate $Q$ is larger than that of the pressure $P$ with the same level of interpolation for all the parameters.

\subsection{Moderate dimensional parametric uncertainty - systemic analysis}

There are many different uncertainties from various sources entering into the coupled hyperbolic system (6), (9) and (10). For a systemic study of their impact to the stochastic solution, we parametrize them with the same signal-to-noise ratio and conduct a global sensitivity analysis by evaluating the main effect (23). More specifically, we consider the following uncertainties:

1. Geometrical parameters: in our one dimensional model, we keep the length of each segment as a deterministic value to retain the geometry of the arterial tree and only consider the reference area $A_{0}$ of the lumen as the uncertain parameter; we incorporate the uncertainty of wall thickness $h$ into the elastic and viscoelastic term;

2. Mathematical and physical parameters: we randomize the blood density $\rho$ and viscosity $\mu$ to account for the convection acceleration and wall friction effects, as well as Young modulus $E$ and characteristic time $T$ to account for the elastic and viscoelastic effects of the arterial wall

*From numerical perspective, we can see that it is sufficient for the first level of interpolation to compute the statistics of both the flow rate $Q$ and the area $A_{0}$ with uncertainty in a small range $(1 / \mathrm{SNR} \approx 10 \%)$ since the error in the second level is not quite different from that in the first level for all the four statistics $\mathbb{E}[Q], \mathbb{S}[Q], \mathbb{E}[P], \mathbb{S}[P]$. In contrast, for a relatively large range of uncertainty for resistance $(1 / \mathrm{SNR} \approx 25.4 \%)$, the first level of interpolation is still sufficient for the first moment (expectation $\mathbb{E}[Q], \mathbb{E}[P]$ ) while the second level of interpolation is needed to have apparently more accurate second moment (standard deviation $\mathbb{S}[Q], \mathbb{S}[P]$ ). When the range of uncertainty is very large, as for Young modulus $(1 / \mathrm{SNR} \approx 53.3 \%)$ with high non-linearity, we need high level of interpolation to evaluate accurately both the expectation and standard deviation as can be seen in Table I that the error between different levels of interpolation is far from each other for all the statistics. 

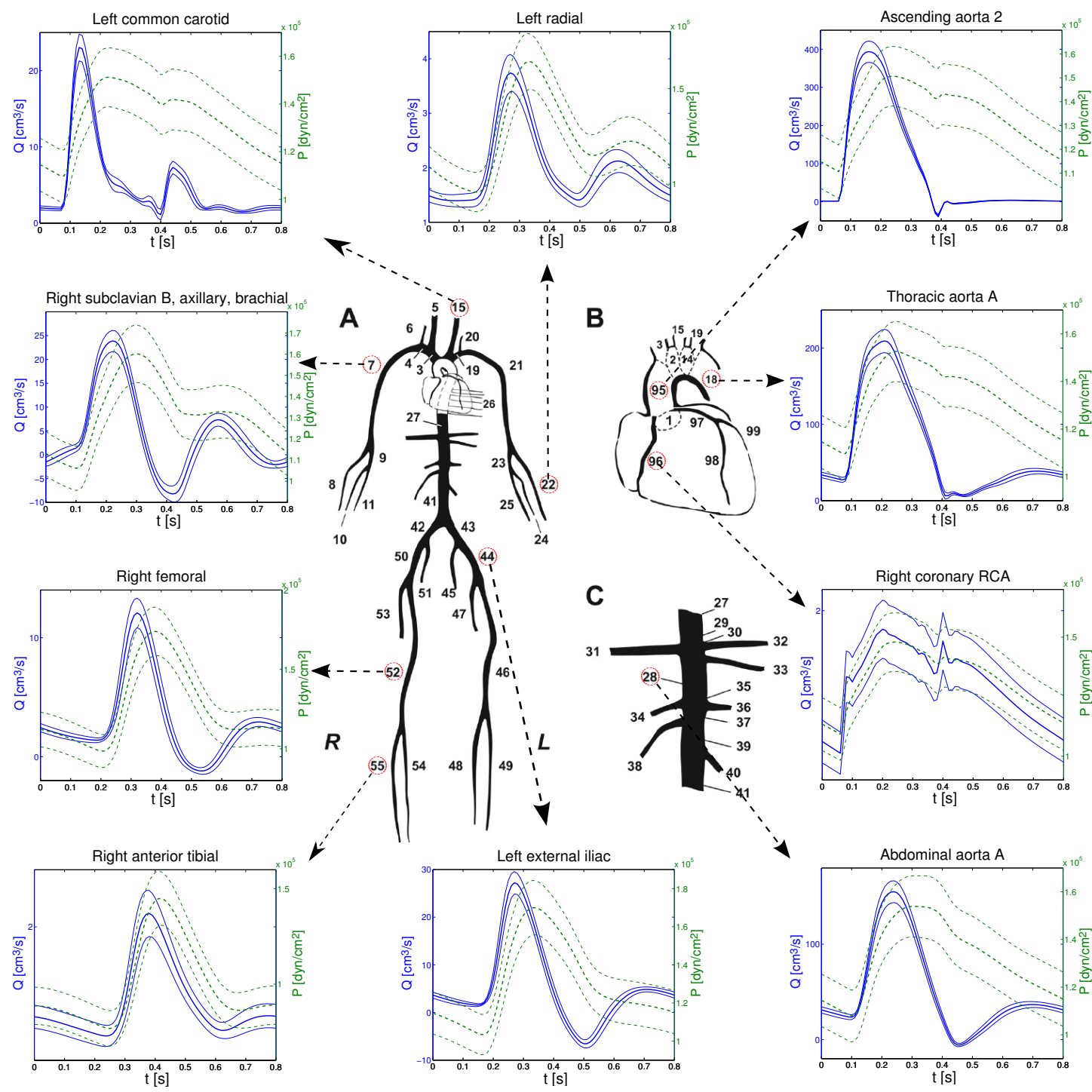

Figure 8. Expectation $\mathbb{E}$ and expectation biased by standard deviation $\mathbb{E}-\mathbb{S}$ and $\mathbb{E}+\mathbb{S}$ of flow rate $Q$ and pressure $P$ at 10 representative locations in the main systemic arterial tree

(Poisson coefficient $\nu$, wall thickness $h$ and the characteristic angle $\phi$ in (5) are incorporated via $E$ and $T$ in each term);

3. Parameters from boundary conditions: the uncertainties arising from the prescribed flow rate $Q$ at the proximal boundary of ascending aorta 1 (1), the resistance $R$ and the capacitance $C$ in the lumped parameter model for the outflow boundary condition are taken into account;

4. External source or force term: we also consider the external pressure $P_{\text {ext }}$ and the venous pressure $P_{v}$ as the sources of uncertainties.

In order to distinguish the impact of these different uncertainties to the solution of the stochastic system (12), we parametrize them with the same signal-to-noise ratio via (13). ${ }^{\dagger}$ Expectation $(\mathbb{E}[\cdot])$

$\dagger^{\dagger}$ For the sake of computational effort, we take $\mu_{e}=0.01, \sigma_{v}=0.1$ to obtain more accurate evaluation of the second statistical moment (e.g. variance, main effect in (23)) with small level of interpolation. Moreover, a small perturbation of these parameters will retain the mathematical property of the hyperbolic system. The Galerkin-Hermite quadrature 

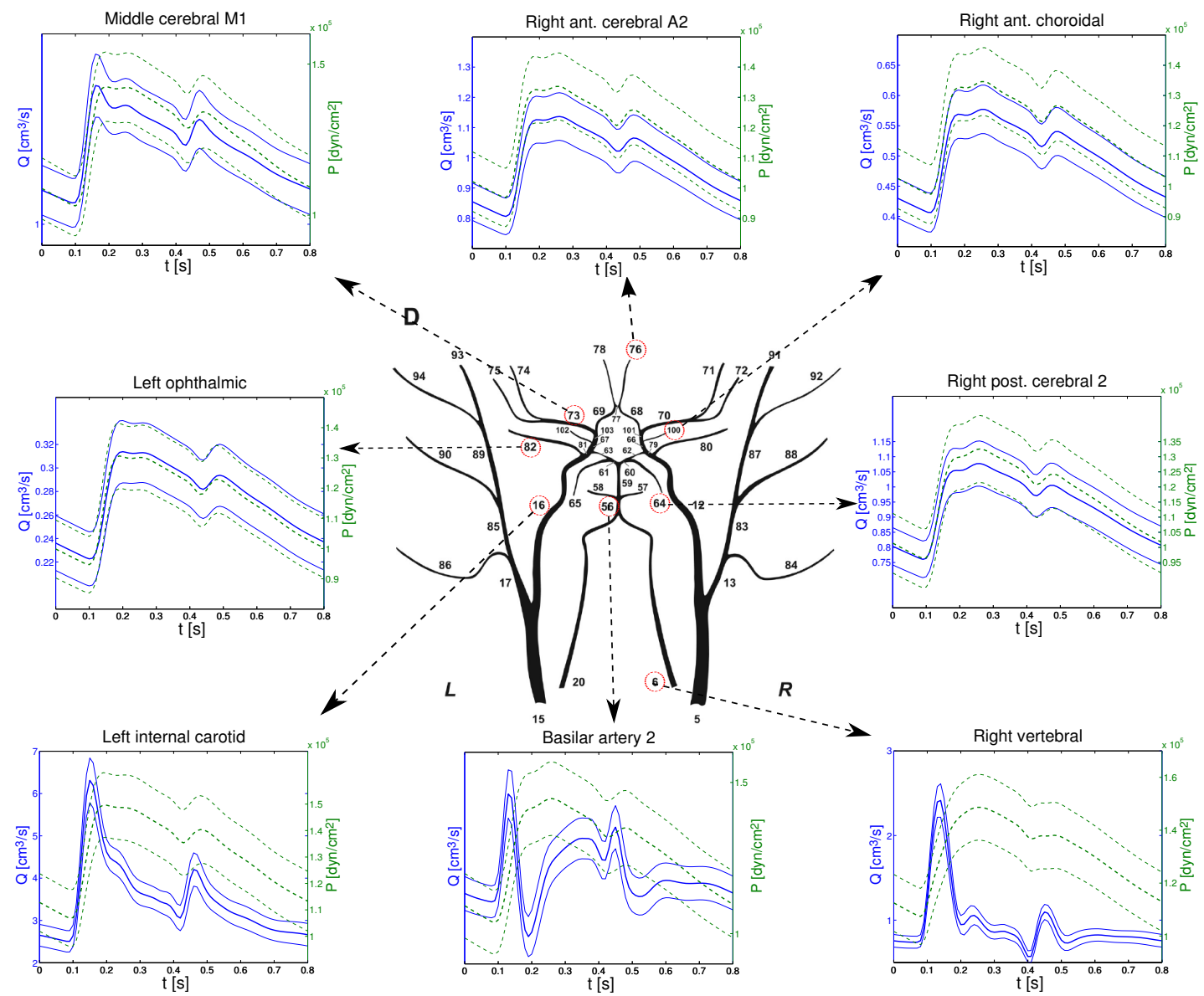

Figure 9. Expectation $\mathbb{E}$ and expectation biased by standard deviation $\mathbb{E}-\mathbb{S}$ and $\mathbb{E}+\mathbb{S}$ of flow rate $Q$ and pressure $P$ at 8 representative locations in the cerebral arterial tree

and expectation biased by standard deviation $(\mathbb{E}[\cdot]-\mathbb{S}[\cdot]$ and $\mathbb{E}[\cdot]+\mathbb{S}[\cdot])$ of the blood flow rate and pressure is shown at the 18 representative locations in Figure 8 for the systemic main arterial tree and Figure 9 for the cerebral arterial tree. From both these two figures, we observe a good overall agreement in both wave shape and amplitude of the blood flow rate and pressure between our simulation and the model prediction in [39], which is validated in high accordance with clinical measurements. In particular, all the shape features of the primary wave and secondary wave of the blood flow from measurement are well captured by our simulations.

Beside the significant similarity between the measurement and the simulation, as both observed in [39] and our work, another important observation from our stochastic simulation is that large variation of the blood flow occurs near the peak of flow wave during the systolic period (see, e.g., ascending aorta 2 (95), thoracic aorta A (18), abdominal aorta A (28)), while the variation of pressure is not that different during the whole heart beat at most of the locations (e.g. left radial (22), left common carotid (15)). Otherwise said, the shape of the flow rate is changed while the

abscissas are used as the collocation nodes for the stochastic collocation method with Smolyak sparse grid interpolation formula (17), where $K=10$ and we use the first $(q-K=1)$ and second $(q-K=2)$ levels of interpolation with 21 and 241 collocation nodes respectively to compute the statistics (20) (22) and sensitivity (23). The relative error as defined in (25) between the two levels of interpolation for the statistics of $\mathbb{E}[Q], \mathbb{S}[Q], \mathbb{E}[P], \mathbb{S}[P]$ are $2.29 \%, 8.69 \%, 0.38 \%, 1.59 \%$, respectively, which are small enough even for the second moment of standard deviation. The small relative error implies that the first level of interpolation is sufficient to evaluate the statistics and also the second moment of main effect (23). We note that all the results we discuss below are evaluated from the second level of interpolation. 


\begin{tabular}{lcccccccccc}
\hline \hline location/parameter & $\rho$ & $\mu$ & $P_{v}$ & $P_{\text {ext }}$ & $Q$ & $R$ & $C$ & $T$ & $E$ & $A_{0}$ \\
Right coronary RCA & 0.181 & 0.003 & 0.114 & 0.000 & 97.059 & 0.735 & 0.000 & 0.000 & 0.051 & 1.750 \\
Ascending aorta 2 & 0.000 & 0.000 & 0.000 & 0.000 & 99.991 & 0.003 & 0.000 & 0.000 & 0.000 & 0.003 \\
Left common carotid & 2.842 & 0.012 & 3.100 & 1.095 & 76.070 & 5.631 & 0.000 & 0.003 & 0.011 & 10.885 \\
Left radial & 0.841 & 0.016 & 0.436 & 0.000 & 95.246 & 1.330 & 0.000 & 0.000 & 0.004 & 1.864 \\
Abdominal aorta A & 0.021 & 0.002 & 0.012 & 0.000 & 99.633 & 0.044 & 0.000 & 0.000 & 0.008 & 0.179 \\
Left external iliac & 0.803 & 0.302 & 0.364 & 0.024 & 86.675 & 3.642 & 0.000 & 0.001 & 0.008 & 8.055 \\
Right anterior tibial & 6.504 & 0.011 & 3.786 & 0.001 & 51.805 & 10.472 & 0.000 & 0.001 & 0.012 & 27.209 \\
Right femoral & 4.572 & 0.022 & 2.895 & 0.001 & 62.812 & 8.657 & 0.000 & 0.001 & 0.002 & 20.725 \\
Thoracic aorta A & 0.069 & 0.001 & 0.029 & 0.000 & 99.312 & 0.094 & 0.000 & 0.000 & 0.005 & 0.395 \\
Right subclavian B & 0.189 & 0.011 & 0.294 & 0.000 & 96.953 & 1.375 & 0.000 & 0.000 & 0.003 & 0.932 \\
\hline Middle cerebral M1 & 4.859 & 0.002 & 3.041 & 0.022 & 55.518 & 5.453 & 0.000 & 0.001 & 0.046 & 30.274 \\
Right ant. cerebral A2 & 0.334 & 0.004 & 0.252 & 0.007 & 98.469 & 0.096 & 0.000 & 0.002 & 0.019 & 0.689 \\
Right ant. choroidal & 0.503 & 0.005 & 0.435 & 0.001 & 95.296 & 0.157 & 0.000 & 0.002 & 0.016 & 3.138 \\
Right post. cerebral 2 & 1.254 & 0.005 & 0.468 & 0.002 & 94.336 & 0.188 & 0.000 & 0.002 & 0.017 & 3.512 \\
Basilar artery 2 & 1.255 & 0.068 & 13.678 & 1.187 & 61.921 & 17.011 & 0.000 & 0.044 & 0.011 & 0.327 \\
Right vertebral & 2.504 & 0.108 & 17.981 & 1.095 & 47.625 & 24.553 & 0.000 & 0.140 & 0.006 & 0.082 \\
Left internal carotid & 3.678 & 0.040 & 4.161 & 2.036 & 70.308 & 6.478 & 0.000 & 0.010 & 0.047 & 12.814 \\
Left ophthalmic & 7.857 & 0.002 & 2.916 & 0.004 & 59.140 & 4.292 & 0.000 & 0.008 & 0.077 & 25.447 \\
\hline \hline
\end{tabular}

Table II. Sensitivity analysis: main effect of different parameters to flow rate $Q$ at 18 locations (\%)

\begin{tabular}{lcccccccccc}
\hline \hline location/parameter & $\rho$ & $\mu$ & $P_{v}$ & $P_{\text {ext }}$ & $Q$ & $R$ & $C$ & $T$ & $E$ & $A_{0}$ \\
Right coronary RCA & 0.192 & 0.089 & 0.126 & 0.054 & 54.096 & 43.612 & 0.000 & 0.000 & 0.049 & 1.755 \\
Ascending aorta 2 & 0.265 & 0.087 & 0.150 & 0.053 & 54.223 & 42.879 & 0.000 & 0.001 & 0.058 & 2.247 \\
Left common carotid & 0.260 & 0.087 & 0.173 & 0.052 & 54.565 & 42.605 & 0.000 & 0.000 & 0.049 & 2.192 \\
Left radial & 0.003 & 0.106 & 0.000 & 0.061 & 51.599 & 48.112 & 0.000 & 0.000 & 0.009 & 0.107 \\
Abdominal aorta A & 0.219 & 0.084 & 0.152 & 0.054 & 54.072 & 43.610 & 0.000 & 0.000 & 0.039 & 1.761 \\
Left external iliac & 0.151 & 0.090 & 0.133 & 0.055 & 54.314 & 43.825 & 0.000 & 0.000 & 0.026 & 1.422 \\
Right anterior tibial & 0.401 & 0.130 & 0.326 & 0.082 & 44.262 & 52.995 & 0.000 & 0.001 & 0.003 & 1.821 \\
Right femoral & 0.093 & 0.095 & 0.052 & 0.058 & 53.570 & 45.369 & 0.000 & 0.000 & 0.015 & 0.746 \\
Thoracic aorta A & 0.259 & 0.087 & 0.166 & 0.053 & 54.490 & 42.687 & 0.000 & 0.000 & 0.051 & 2.187 \\
Right subclavian B & 0.220 & 0.088 & 0.200 & 0.053 & 54.998 & 42.514 & 0.000 & 0.000 & 0.030 & 1.917 \\
\hline Middle cerebral M1 & 0.088 & 0.083 & 0.162 & 0.135 & 54.319 & 44.367 & 0.000 & 0.000 & 0.019 & 0.880 \\
Right ant. cerebral A2 & 0.020 & 0.095 & 0.113 & 0.089 & 51.800 & 47.909 & 0.000 & 0.001 & 0.012 & 0.006 \\
Right ant. choroidal & 0.023 & 0.098 & 0.109 & 0.070 & 52.167 & 47.489 & 0.000 & 0.001 & 0.012 & 0.013 \\
Right post. cerebral 2 & 0.049 & 0.102 & 0.116 & 0.076 & 51.354 & 48.311 & 0.000 & 0.001 & 0.012 & 0.026 \\
Basilar artery 2 & 0.064 & 0.096 & 0.058 & 0.069 & 53.374 & 45.587 & 0.000 & 0.000 & 0.023 & 0.733 \\
Right vertebral & 0.183 & 0.089 & 0.148 & 0.058 & 54.470 & 43.431 & 0.000 & 0.000 & 0.033 & 1.598 \\
Left internal carotid & 0.184 & 0.091 & 0.144 & 0.047 & 54.460 & 43.368 & 0.000 & 0.000 & 0.033 & 1.676 \\
Left ophthalmic & 0.057 & 0.113 & 0.249 & 0.046 & 53.118 & 46.493 & 0.000 & 0.000 & 0.014 & 0.002 \\
\hline \hline
\end{tabular}

Table III. Sensitivity analysis: main effect of different parameters to pressure $P$ at 18 locations (\%)

shape of the pressure remains almost the same with its mean value changed. This observation is well verified from the measurements with also similar amplitude of variation (see Fig. 4 and 5 in [39] for the corresponding specific locations) and implies that the difference of blood flow and pressure wave propagation among different people arises probably from the kind of uncertainties we are investigating.

In order to quantify systemically the impact of uncertainties of different parameters, we compute the main effect of each parameter to the blood flow rate and pressure averaged over one heart beat via the global sensitivity index in formula (23), with the results (value in percent (\%)) shown in Table II for flow rate and III for pressure, from which we have the following observations:

1. From the main effect values for both flow rate and pressure, we see that with the same signal-to-noise ratio, the uncertainty arising from the imposed inflow rate $Q$ at the aortic root dominates all the other uncertainties as for the overall impact to the simulation results. This result is compliant with the physiological fact that under different working effort, e.g. running 

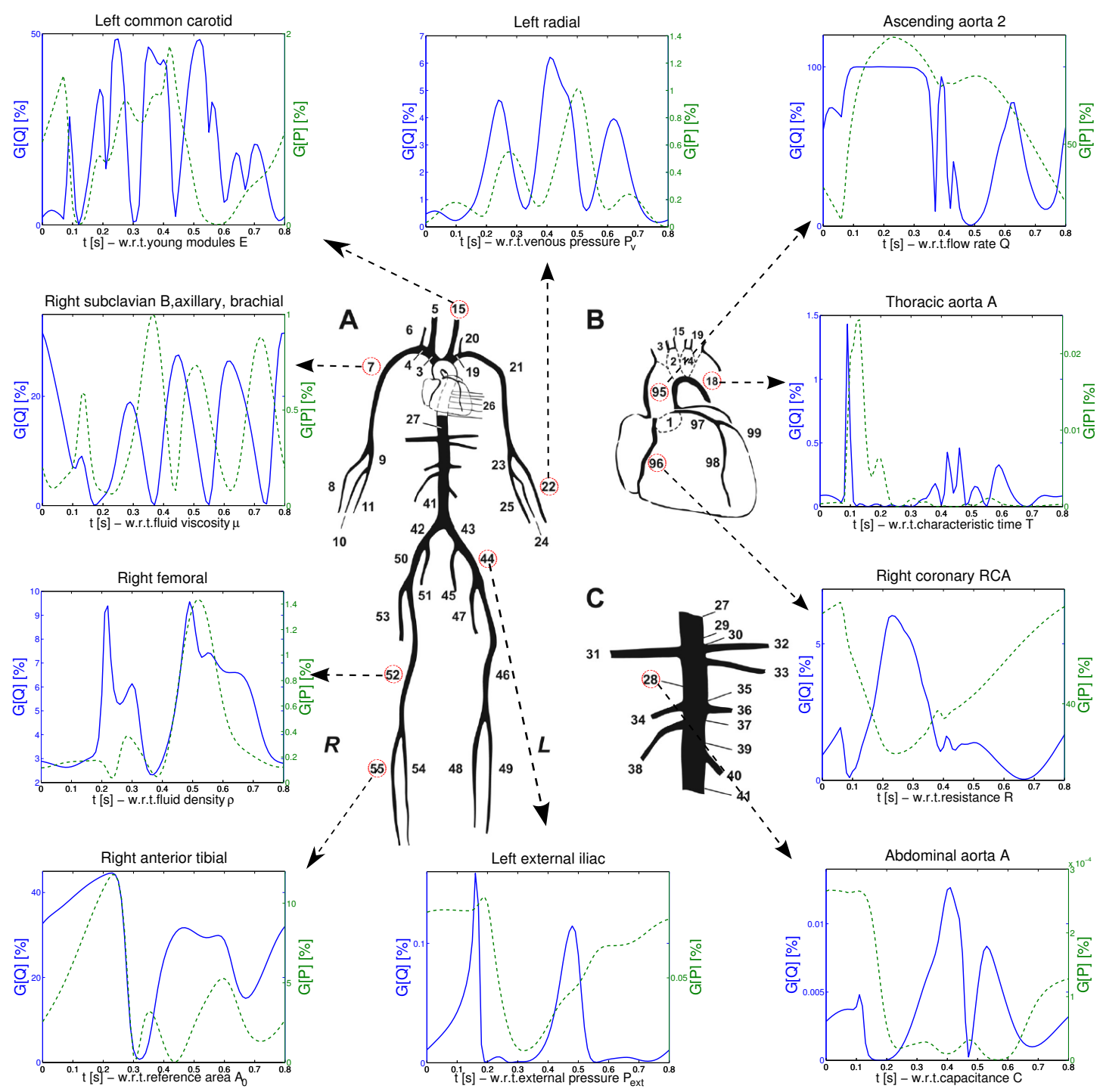

B
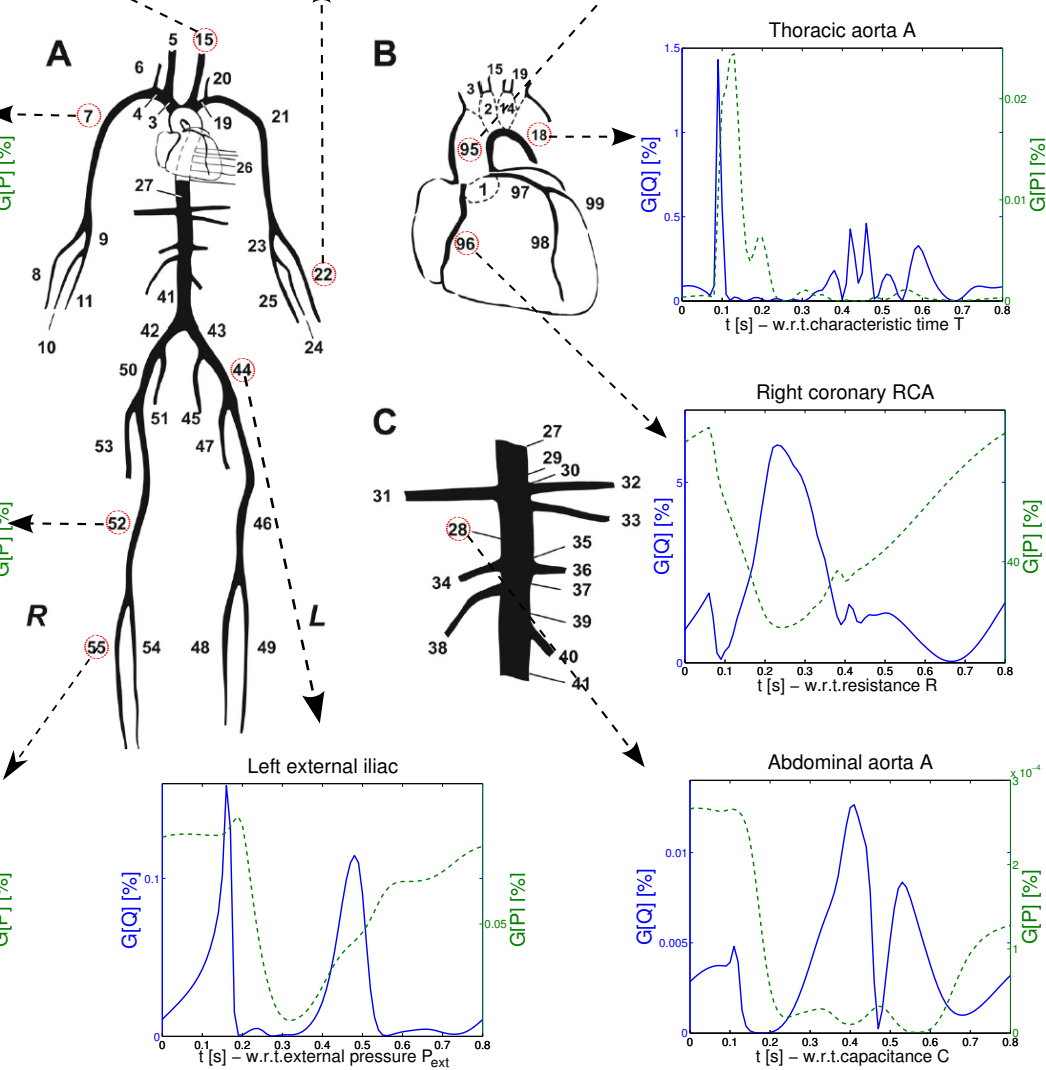

Figure 10. Time dependent main effect (global sensitivity index defined in (23)) over one heart beat at 10 representative locations in the main systemic arterial tree

or sleeping, the inflow rate not only spans in a large range but also plays a major role in affecting the flow rate and pressure in the global arterial tree [47]. Moreover, this uncertainty displays distinct impact to the flow rate at different locations: in the systemic main arterial tree, the impact is large near the heart (e.g. $99.99 \%$ at ascending aorta 2 ) and relatively small at peripheral vessels (e.g. $51.80 \%$ at right anterior tibial); however, the conclusion becomes opposite in the cerebral arterial tree (e.g. $47.63 \%$ at right vertebral and $98.47 \%$ at right ant. cerebral A2).

2. At the peripheral vessels in the systemic main arterial tree, variation from the reference area also plays an evident role in affecting the blood flow rate (e.g. $27.21 \%$ at right anterior tibial, $20.73 \%$ at right femoral), which implies that some peripheral arterial diseases may result from the variation of the reference cross section area, such as atherosclerosis or stenosis [34]. A relatively large impact of uncertainty from the reference area can also be observed at the left common carotid (10.88\%) and right coronary RCA (1.75\%), which coincides with some 
pathological implications that dysfunctions of the brain (e.g. stroke) and the heart (e.g. heart attack) are due to the blockage of blood flow in the common carotid and coronary arteries [3].

3. In contrast, the variation of the inflow rate does not have so different effects on the pressure at different locations, staying between $50 \%-55 \%$ for both the systemic main arterial tree and the cerebral arterial tree with an exception $44.3 \%$ (see Table III) from the most distal arterial segment right anterior tibial. In fact, most of the uncertainties display a relatively larger variation at different locations for the flow rate than for the pressure, as can be observed quite evidently from Table II and III. It is interesting to point out that the uncertainty arising from the resistance at the terminal boundaries has a major impact to pressure, over $40 \%$ at all the representative locations. This implies that the inter-individual variability of the pressure may arise from different structure of terminal arterial network and a more detailed modeling of the arterial network at the terminal boundaries would help to improve the simulation accuracy of the pressure.

4. As for the uncertainty arising from physical parameters $\rho, \mu, C, T, E$ and prescribed pressure $P_{v}, P_{e x t}$, they are not so important as the parameters analyzed above for both flow rate and pressure. We remark that the analysis is under the same signal-to-noise ratio for all parameters. However, the fact is that some of the parameters are easy to measure with high accuracy in practice, e.g. inflow rate, reference area, while some others, e.g. young modulus, are more difficult to measure, thus they are affected by high noise potentially leading to large variations on the simulation results.
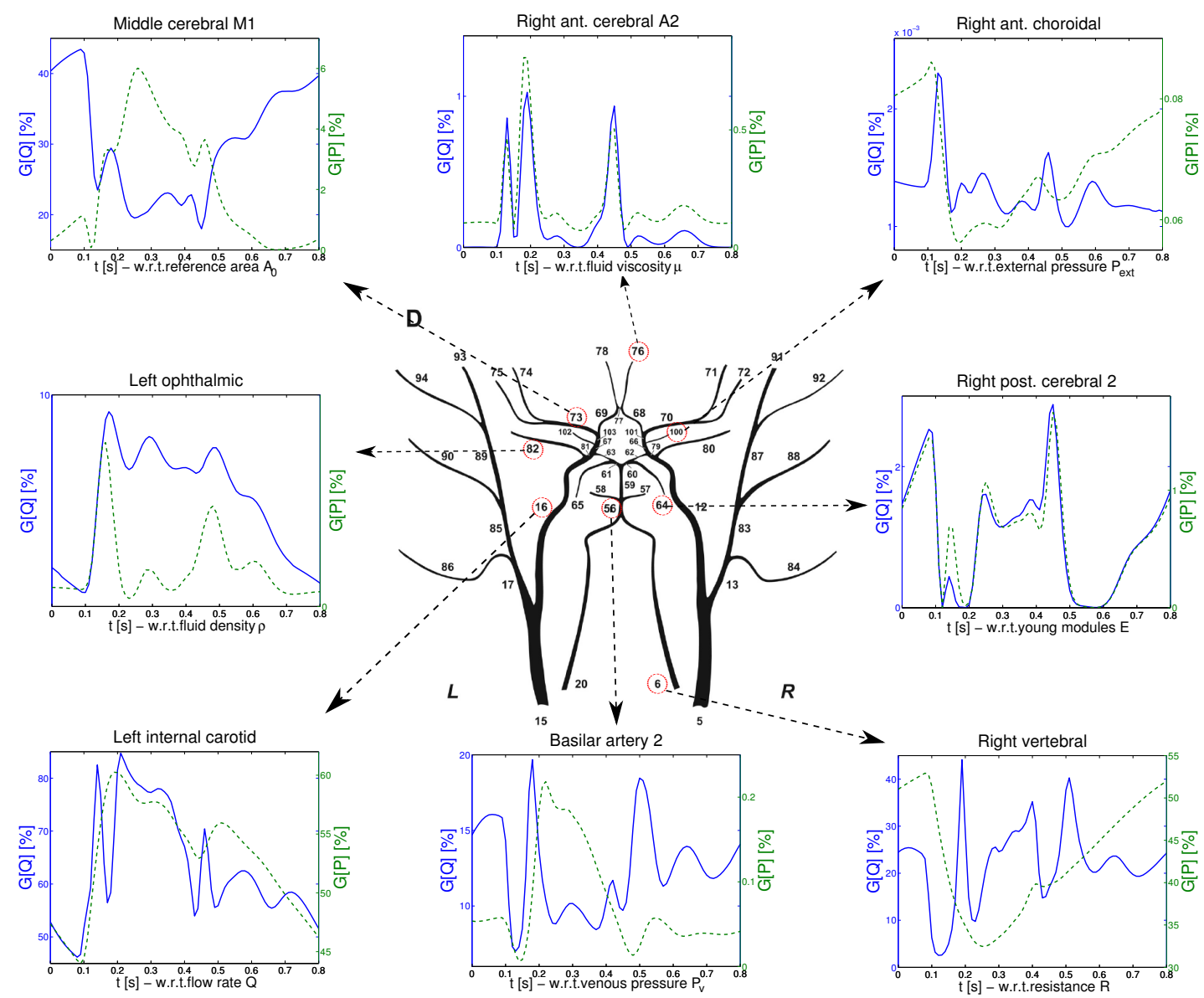

Figure 11. Time dependent main effect (global sensitivity index defined in (23)) over one heart beat at 8 representative locations in the cerebral arterial tree 
In order to have a closer look at the sensitivity of flow rate and pressure with respect to all of the uncertainties at different time of one heart beat, we compute the variation in time of the sensitivity (time dependent main effect) of different sources of uncertainties at different locations. We pick the most important source of uncertainty in general according to Table II, e.g. imposed heart flow rate $Q$, at the most evident location, e.g. ascending aorta 2 (95), and then the second most important source at the second most evident location and so on. Following this order we plot the Figure 10 and 11 for the time dependent main effect, which delivers more information about the uncertainty impact at different time. More specifically, we can draw the following conclusions:

1. The main effect (global sensitivity in time) of most of the parametric uncertainties varies in a large range within one heart beat. The variation of the main effect of some uncertainties is quite different or even opposite for flow rate and pressure in some local time region, e.g. resistance $R$ at right coronary RCA (96) or reference area $A_{0}$ at middle cerebral M1 (73), and changes in a similar way for some other uncertainties, e.g. Young modulus $E$ at Right post.cerebral 2 (64) or venous pressure $P_{v}$ at left radial (22). In general, even though some uncertainties dominate the others, as observed from the averaged main effect in Table II and III, from the variation of the main effect in time we can see that quite a few uncertainties play a near important role in an oscillating way. This suggests that when considering impact of uncertainty at local time, it would be misleading to overemphasize some uncertainties and neglect some others.

2. The large variation of the main effect occurs at some common time, especially at the beginning of systolic period and end of the diastolic period, e.g. flow rate $Q$ at ascending aorta 2 (95) or characteristic time $T$ at thoracic aorta A (18), or at the time of maximum or minimum flow rate during the systolic and diastolic periods, e.g. fluid density $\rho$ at left ophthalmic (82) or fluid viscosity $\mu$ at right ant. cerebral A2 (76). Moreover, the evident main effect of most of the uncertainties span in a relatively large time range over one heart beat, e.g. flow rate $Q$ at ascending aorta 2 (95) or Young modulus $E$ at left common carotid (15), while for some other few uncertainties, it restricts at a small local time with peaks, e.g. characteristic time $T$ at thoracic aorta A (18) or fluid viscosity $\mu$ at right ant. cerebral A2 (76).

\subsection{High dimensional parametric uncertainty - differentiated analysis}

For systemic quantification of the impact of different uncertainties, it is reasonable to use the same probability distribution for one uncertainty in the global arterial network. However, in order to quantify some uncertainty arising from the same source but at different locations, it is more realistic to perform differentiated analysis by employing independent random variables to characterize the uncertainty at each of the location. In this section, we take two of the influential uncertainties for the blood wave propagation (see Table III), resistance $R$ at the 47 different distal boundaries and cross section reference area $A_{0}$ in the 103 different arterial segments of the schematic representation of the human arterial tree in Figure 3, and assign independent random variables to each of them at different locations to study locally the impact of different uncertainties.

We assume that the resistance at the $m$ th distal boundary is randomized by (13) as

$$
R_{m}(\omega)=\exp \left(\mu_{e}+\sigma_{v} Y_{m}(\omega)\right) R_{m}, 1 \leq m \leq M
$$

where $Y_{m}, 1 \leq m \leq M$ are independent random variables obeying standard distribution.

The largest five values of the main effect ${ }^{\S}$ and their total weight (in percent (\%)) is shown in Table IV and V, from which the following physiological implications can be drawn:

\footnotetext{
$\S$ We choose $\mu_{e}=0.01, \sigma_{v}=0.1$ for the sake of computational effort and accurate evaluation of the statistics and sensitivity with small level of interpolation. By the main effect formula (23) and Smolyak sparse grid collocation method (17) with the first level of interpolation $q-K=1$, we compute main sensitivity at the 18 representative locations with respect to each random resistance at the 47 distal boundaries.
} 
1. In general, the largest impact to flow rate comes from the uncertainty of resistance at the nearest distal boundaries and it dominates the impact of resistance variation from all the other boundaries, e.g. the impact to right anterior tibial (92.14\%) comes from its distal boundary.

2. In particular, the largest impact of the uncertainty of resistance to flow rate at the ascending aorta 2 comes from the coronary arteries $(99,98,96)$, which implies that strong resistance or even blockage of the coronary arteries would potentially lead to heart attack or heart failure as expected.

3. As for the impact to pressure, the largest values come mostly from the uncertainty of resistance at the distal boundaries of abdominal aorta branches along the ascending aorta $(34,36,38$, 33 ), no matter where the location of the pressure is considered. This indicates that resistance at these locations have a major influence to the global pressure and a more detailed modeling of the branch arteries is needed to improve the simulation precision.

\begin{tabular}{lcccccc}
\hline \hline location/order & 1st & 2nd & 3rd & 4th & 5th & $1-5$ \\
Right coronary RCA & $98.010(96)$ & $0.579(34)$ & $0.385(36)$ & $0.383(38)$ & $0.288(26)$ & 99.645 \\
Ascending aorta 2 & $33.864(99)$ & $22.409(98)$ & $20.864(96)$ & $6.975(34)$ & $4.649(36)$ & 88.762 \\
Left common carotid & $16.781(34)$ & $11.166(36)$ & $11.142(38)$ & $9.760(78)$ & $8.225(26)$ & 57.074 \\
Left radial & $96.782(22)$ & $0.923(34)$ & $0.613(36)$ & $0.612(38)$ & $0.459(26)$ & 99.389 \\
Abdominal aorta A & $35.757(34)$ & $23.834(36)$ & $23.802(38)$ & $6.727(26)$ & $2.027(33)$ & 92.147 \\
Left external iliac & $64.780(47)$ & $14.886(48)$ & $4.873(34)$ & $4.369(49)$ & $3.291(38)$ & 92.198 \\
Right anterior tibial & $92.143(55)$ & $2.066(34)$ & $1.403(38)$ & $1.395(36)$ & $1.044(54)$ & 98.051 \\
Right femoral & $65.185(54)$ & $19.086(55)$ & $4.749(34)$ & $3.213(38)$ & $3.200(36)$ & 95.434 \\
Thoracic aorta A & $28.948(34)$ & $19.304(36)$ & $19.281(38)$ & $13.910(26)$ & $4.030(33)$ & 85.472 \\
Right subclavian B & $47.514(8)$ & $38.477(11)$ & $4.160(34)$ & $2.767(36)$ & $2.761(38)$ & 95.680 \\
\hline Middle cerebral M1 & $45.862(75)$ & $44.636(74)$ & $2.839(34)$ & $1.886(36)$ & $1.881(38)$ & 97.103 \\
Right ant. cerebral A2 & $98.004(76)$ & $0.569(34)$ & $0.378(36)$ & $0.377(38)$ & $0.283(26)$ & 99.610 \\
Right ant. choroidal & $97.686(100)$ & $0.670(34)$ & $0.445(36)$ & $0.443(38)$ & $0.334(26)$ & 99.577 \\
Right post. cerebral 2 & $97.666(64)$ & $0.670(34)$ & $0.445(36)$ & $0.444(38)$ & $0.334(26)$ & 99.560 \\
Basilar artery 2 & $34.116(65)$ & $34.053(64)$ & $12.039(58)$ & $12.039(57)$ & $1.273(34)$ & 93.520 \\
Right vertebral & $34.364(65)$ & $34.298(64)$ & $12.124(58)$ & $12.124(57)$ & $1.166(78)$ & 94.075 \\
Left internal carotid & $23.796(78)$ & $10.593(102)$ & $10.019(34)$ & $8.436(75)$ & $8.303(76)$ & 61.147 \\
Left ophthalmic & $95.852(82)$ & $1.232(34)$ & $0.819(36)$ & $0.817(38)$ & $0.615(26)$ & 99.336 \\
\hline \hline
\end{tabular}

Table IV. The largest five values and their summation of main effect (value in percent (\%)) of flow rate with respect to resistance from their corresponding boundaries in bracket $(\cdot)$ at 18 locations

\begin{tabular}{lcccccc}
\hline \hline location/order & 1st & 2nd & 3rd & 4th & 5th & $1-5$ \\
Right coronary RCA & 29.782(34) & $19.783(36)$ & $19.725(38)$ & $14.823(26)$ & $4.135(33)$ & 88.248 \\
Ascending aorta 2 & $30.395(34)$ & $20.190(36)$ & $20.131(38)$ & $15.127(26)$ & $4.219(33)$ & 90.063 \\
Left common carotid & $30.390(34)$ & $20.186(36)$ & $20.127(38)$ & $15.129(26)$ & $4.219(33)$ & 90.051 \\
Left radial & $24.430(22)$ & $22.267(34)$ & $14.790(36)$ & $14.747(38)$ & $11.115(26)$ & 87.350 \\
Abdominal aorta A & $30.959(34)$ & $20.557(36)$ & $20.491(38)$ & $14.091(26)$ & $4.251(33)$ & 90.350 \\
Left external iliac & $30.371(34)$ & $20.597(38)$ & $20.478(36)$ & $13.650(26)$ & $4.133(33)$ & 89.229 \\
Right anterior tibial & $53.200(55)$ & $12.236(34)$ & $8.317(38)$ & $8.264(36)$ & $6.540(54)$ & 88.556 \\
Right femoral & $29.180(34)$ & $19.806(38)$ & $19.688(36)$ & $13.052(26)$ & $3.972(33)$ & 85.698 \\
Thoracic aorta A & $30.403(34)$ & $20.194(36)$ & $20.134(38)$ & $15.138(26)$ & $4.221(33)$ & 90.091 \\
Right subclavian B & $30.117(34)$ & $20.007(36)$ & $19.952(38)$ & $14.996(26)$ & $4.179(33)$ & 89.252 \\
\hline Middle cerebral M1 & $29.744(34)$ & $19.757(36)$ & $19.699(38)$ & $14.814(26)$ & $4.129(33)$ & 88.143 \\
Right ant. cerebral A2 & $24.067(76)$ & $22.659(34)$ & $15.050(36)$ & $15.005(38)$ & $11.286(26)$ & 88.067 \\
Right ant. choroidal & $23.451(34)$ & $21.853(100)$ & $15.578(36)$ & $15.532(38)$ & $11.681(26)$ & 88.094 \\
Right post. cerebral 2 & $27.227(64)$ & $21.695(34)$ & $14.411(36)$ & $14.369(38)$ & $10.805(26)$ & 88.506 \\
Basilar artery 2 & $29.461(34)$ & $19.569(36)$ & $19.512(38)$ & $14.668(26)$ & $4.090(33)$ & 87.300 \\
Right vertebral & $30.179(34)$ & $20.047(36)$ & $19.989(38)$ & $15.023(26)$ & $4.190(33)$ & 89.429 \\
Left internal carotid & $30.228(34)$ & $20.079(36)$ & $20.020(38)$ & $15.053(26)$ & $4.197(33)$ & 89.576 \\
Left ophthalmic & $24.635(34)$ & $17.590(82)$ & $16.356(36)$ & $16.308(38)$ & $12.280(26)$ & 87.168 \\
\hline \hline
\end{tabular}

Table V. The largest five values and their summation of main effect (value in percent (\%)) of pressure with respect to resistance from their corresponding boundaries in bracket $(\cdot)$ at 18 locations 
As for the uncertainty quantification with respect to reference area $A_{0}$, we take the same numerical design as for the resistance, leading to 103 independent random variables. The main effect results are shown in Table VI for flow rate and VII for pressure, from which we may have the following observations:

1. In general, the most important uncertainty for the flow rate at the representative locations comes from the location itself or the few nearest arterial segments. For instance, the main effect with the largest value $98.5 \%$ at left ophthalmic (82) in the cerebral arterial tree is due to the uncertainty of the reference area of the arterial segment itself.

2. The largest values of the main effect for the pressure concentrate again at the few locations $(27,95,18)$, which are very close to the heart, i.e. a small change of the cross section area of the large arteries near the heart leads to relatively large variation of pressure wave propagation in the global arterial network, which implies that some arterial diseases such as aorta atherosclerosis or the equivalent aorta stiffening may result in severe change of the global blood pressure.

3. An interesting observation is that the main effect of the flow rate at the abdominal aorta A (28) distributes rather dispersedly than concentrated in a small region, i.e. coming from the uncertainty of the reference area not only near the heart (ascending aorta 2 (95)) but also from the four longest peripheral arterial segments - the arms $(7,21)$ and legs $(52,46)$ as well as other regions. These many influential sources might provide an environment to develop aneurysms with high probability, which could also be observed in the basilar artery 2 (56), see Table VI.

\begin{tabular}{|c|c|c|c|c|c|c|}
\hline location/order & 1 st & 2nd & $3 \mathrm{rd}$ & 4th & 5th & $1-5$ \\
\hline Right coronary RCA & $45.498(96)$ & $13.333(27)$ & $9.516(95)$ & $4.743(18)$ & $4.091(21)$ & 77.181 \\
\hline Ascending aorta 2 & $77.616(95)$ & $7.039(1)$ & $5.138(98)$ & $1.907(27)$ & $1.225(46)$ & 92.925 \\
\hline Left common carotid & $58.289(16)$ & $10.457(12)$ & $7.408(15)$ & $3.479(69)$ & $3.311(68)$ & 82.943 \\
\hline Left radial & $89.126(22)$ & $2.912(21)$ & $2.443(27)$ & $1.362(95)$ & $0.994(7)$ & 96.837 \\
\hline Abdominal aorta A & $15.811(95)$ & $10.886(7)$ & $10.786(21)$ & $8.570(52)$ & $8.570(46)$ & 54.623 \\
\hline Left external iliac & $46.320(46)$ & $22.054(49)$ & $19.212(48)$ & 4.457 (44) & $1.769(47)$ & 93.811 \\
\hline Right anterior tibial & $92.292(55)$ & $6.218(52)$ & $0.329(54)$ & $0.318(95)$ & $0.181(7)$ & 99.338 \\
\hline Right femoral & $33.939(55)$ & $32.890(52)$ & $29.903(54)$ & $0.629(7)$ & $0.566(95)$ & 97.927 \\
\hline Thoracic aorta A & $25.131(95)$ & $13.627(21)$ & $12.261(7)$ & 8.885 (14) & $4.138(15)$ & 64.042 \\
\hline Right subclavian B & $32.805(11)$ & $29.928(7)$ & $26.728(8)$ & $2.912(21)$ & $1.692(27)$ & 94.065 \\
\hline Middle cerebral M1 & $48.828(74)$ & $48.447(75)$ & $0.820(16)$ & $0.479(27)$ & $0.325(95)$ & 98.898 \\
\hline Right ant. cerebral A2 & $75.245(76)$ & $4.403(27)$ & $3.530(12)$ & $3.005(95)$ & $2.095(68)$ & 88.277 \\
\hline Right ant. choroidal & $91.213(100)$ & $2.504(12)$ & $1.562(27)$ & $1.069(95)$ & $0.604(9)$ & 96.953 \\
\hline Right post. cerebral 2 & $91.862(64)$ & $1.878(27)$ & $1.287(95)$ & $0.743(21)$ & $0.670(18)$ & 96.441 \\
\hline Basilar artery 2 & $27.970(9)$ & $18.040(20)$ & $15.745(16)$ & $15.338(6)$ & $15.088(12)$ & 92.181 \\
\hline Right vertebral & $63.532(6)$ & $19.705(20)$ & $6.048(9)$ & $3.454(16)$ & $3.319(12)$ & 96.058 \\
\hline Left internal carotid & $62.295(16)$ & $11.773(12)$ & $4.017(69)$ & $3.794(68)$ & $3.604(74)$ & 85.483 \\
\hline Left ophthalmic & $98.458(82)$ & $0.491(16)$ & $0.267(27)$ & $0.180(95)$ & $0.096(9)$ & 99.492 \\
\hline
\end{tabular}

Table VI. The largest five values and their summation of main effect (value in percent (\%)) of flow rate with respect to reference area from their corresponding boundaries in bracket $(\cdot)$ at 18 locations

\section{CONCLUDING REMARKS}

In this work we presented a general framework for simulation-based uncertainty quantification in the human arterial network. We identified the main uncertainties arising from various sources and characterized them by random variables obeying certain specific probability distributions. Based on a one dimensional deterministic fluid structure interaction model (a system of nonlinear hyperbolic equation), we introduced the stochastic model by incorporating many different kinds of uncertainties into blood flow in arteries and its interaction with arterial walls in a human arterial network. 


\begin{tabular}{lcccccc}
\hline \hline location/order & 1st & 2nd & 3rd & 4th & 5th & $1-5$ \\
Right coronary RCA & $22.474(27)$ & $16.051(95)$ & $8.012(18)$ & $7.522(96)$ & $7.005(21)$ & 61.064 \\
Ascending aorta 2 & $24.309(27)$ & $16.979(95)$ & $8.655(18)$ & $7.586(21)$ & $7.217(7)$ & 64.745 \\
Left common carotid & $24.982(27)$ & $16.981(95)$ & $8.859(18)$ & $7.841(21)$ & $7.411(7)$ & 66.074 \\
Left radial & $44.965(22)$ & $13.821(21)$ & $12.215(27)$ & $7.231(95)$ & $3.974(7)$ & 82.208 \\
Abdominal aorta A & $22.207(95)$ & $10.407(21)$ & $9.691(7)$ & $8.295(18)$ & $8.071(9)$ & 58.672 \\
Left external iliac & $23.361(95)$ & $12.058(21)$ & $11.785(7)$ & $9.017(18)$ & $7.659(14)$ & 63.880 \\
Right anterior tibial & $50.087(55)$ & $40.073(52)$ & $2.088(95)$ & $2.007(54)$ & $1.093(7)$ & 95.347 \\
Right femoral & $27.526(52)$ & $15.743(95)$ & $8.293(7)$ & $8.136(21)$ & $6.052(18)$ & 65.751 \\
Thoracic aorta A & $25.402(27)$ & $17.153(95)$ & $8.025(21)$ & $7.539(7)$ & $7.500(18)$ & 65.618 \\
Right subclavian B & $26.990(27)$ & $17.276(95)$ & $9.161(18)$ & $8.645(21)$ & $6.995(9)$ & 69.067 \\
\hline Middle cerebral M1 & $28.971(16)$ & $16.817(27)$ & $11.438(95)$ & $6.024(18)$ & $5.665(9)$ & 68.915 \\
Right ant. cerebral A2 & $46.297(76)$ & $9.406(27)$ & $7.563(12)$ & $6.424(95)$ & $4.490(68)$ & 74.180 \\
Right ant choroidal & $59.741(100)$ & $11.213(12)$ & $6.968(27)$ & $4.773(95)$ & $2.701(9)$ & 85.396 \\
Right post. cerebral 2 & $63.271(64)$ & $8.232(27)$ & $5.648(95)$ & $3.260(21)$ & $2.936(18)$ & 83.346 \\
Basilar artery 2 & $21.416(27)$ & $14.728(95)$ & $8.760(21)$ & $7.740(7)$ & $7.629(18)$ & 60.272 \\
Right vertebral & $23.829(27)$ & $16.475(95)$ & $10.724(7)$ & $8.473(18)$ & $8.384(21)$ & 67.885 \\
Left internal carotid & $24.066(27)$ & $16.355(95)$ & $8.579(18)$ & $7.691(21)$ & $7.295(7)$ & 63.987 \\
Left ophthalmic & $62.446(82)$ & $11.727(16)$ & $6.309(27)$ & $4.281(95)$ & $2.287(9)$ & 87.050 \\
\hline \hline
\end{tabular}

Table VII. The largest five values and their summation of main effect (value in percent (\%)) of pressure with respect to reference area from their corresponding boundaries in bracket $(\cdot)$ at 18 locations

One of the most efficient stochastic computational methods - the stochastic collocation method with Smolyak sparse grid - was applied to discretize the coupled stochastic hyperbolic system in stochastic space. In order to study the impact of these uncertainties on the blood flow and pressure wave propagation, we provided statistical and sensitivity analysis by evaluating and interpreting the expectation, standard deviation as well as the main effect accounting for global sensitivity. Our method has been applied to analyze three different kinds of experiments with physiological and pathological implications: 1, one dimensional parametric uncertainty to examine the stochastic regularity and nonlinearity of the solution with respect to different uncertainties as well as the convergence analysis of the sparse grid stochastic collocation method; 2, moderate dimensional parametric uncertainty to make a systemic study of the impact of different uncertainties on the blood flow and pressure in some representative locations; 3, high dimensional parametric uncertainty with differentiated analysis to highlight the correlation of different local arterial segments for the impact of some uncertainty in the blood flow and pressure wave propagation. These experiments provide the first generic simulation-based uncertainty quantification in human arterial network with uncertainties arising from various sources.

There are several limitations in our work that we would like to point out: 1, the probability distribution we used was assumed to be log-normal type independent of time and space, yet for more physiological uncertainty quantification, we need to calibrate realistic probability distribution according to more specific medical data; 2 , the degree of uncertainty is restricted to be small with noise-to-signal around $10 \%$ in order to have more accurate evaluation of statistics with the first level of interpolation for high dimensional problems constrained by computational effort; 3 , there are still many other facts that we didn't consider in our model, the arterial network being not as complete as possible, large arteries with strong local flow field not being suitably described (the use of a fully 3D model would be more appropriate in this case), etc. More research effort is needed to address these limitations, for instance, how to apply the optimal control or optimization strategy to calibrate the probability distribution for different uncertainties [16], how to reduce the stochastic computational effort and increase the accuracy of the numerical solutions by taking full advantage of a small sample of solutions and the structure of the stochastic manifold with reduced basis method [15].

Acknowledgement: The authors thank Dr. A.C.I. Malossi to help us for using the deterministic solver of the coupled one dimensional fluid structure interaction model in LifeV library. This work is partially supported by Swiss National Science Foundation under grant N.200021_141034 and 
the MATHCARD project ERC-2008-AdG-227058 http://www.mathcard.eu/. G. Rozza acknowledges the excellence grant NOFYSAS of SISSA.

\section{REFERENCES}

1. LifeV Project, http://www.lifev.org/.

2. D. Ambrosi, A. Quarteroni, and G. Rozza. Modelling of Physiological Flows. Springer, Series MS\&A, 2011.

3. American Heart Association. Heart and stroke facts. The Association, 1991.

4. I. Babuška, F. Nobile, and R. Tempone. A stochastic collocation method for elliptic partial differential equations with random input data. SIAM Journal on Numerical Analysis, 45(3):1005-1034, 2007.

5. I. Babuška, F. Nobile, and R. Tempone. Reliability of computational science. Numerical Methods for Partial Differential Equations, 23(4):753-784, 2007.

6. I. Babuška, F. Nobile, and R. Tempone. A stochastic collocation method for elliptic partial differential equations with random input data. SIAM review, 52(3):317, 2010.

7. I. Babuška, R. Tempone, and G.E. Zouraris. Galerkin finite element approximations of stochastic elliptic partial differential equations. SIAM Journal on Numerical Analysis, 42(2):800-825, 2005.

8. S. Badia, A. Quaini, and A. Quarteroni. Modular vs. non-modular preconditioners for fluid-structure systems with large added-mass effect. Computer Methods in Applied Mechanics and Engineering, 197(49):4216-4232, 2008.

9. S. Badia, A. Quaini, and A. Quarteroni. Splitting methods based on algebraic factorization for fluid-structure interaction. SIAM Journal on Scientific Computing, 30(4):1778-1805, 2008.

10. S. Boyaval, C. Le Bris, T. Lelièvre, Y. Maday, N.C. Nguyen, and A.T. Patera. Reduced basis techniques for stochastic problems. Archives of Computational Methods in Engineering, 17:435-454, 2010.

11. J.T. Bushberg. The essential physics of medical imaging. Williams \& Wilkins, 2002.

12. G.T. Buzzard and D. Xiu. Variance-based global sensitivity analysis via sparse-grid interpolation and cubature. Communications in Computational Physics, 9:542-67, 2011.

13. C. Canuto, MY Hussaini, A. Quarteroni, and TA Zang. Spectral Methods: Fundamentals in Single Domains. Springer, 2006.

14. D. Chapelle, M. Fernández, J.F. Gerbeau, P. Moireau, J. Sainte-Marie, and N. Zemzemi. Numerical simulation of the electromechanical activity of the heart. Functional Imaging and Modeling of the Heart, 5528:357-365, 2009.

15. P. Chen, A. Quarteroni, and G. Rozza. Comparison of reduced basis method and collocation method for stochastic elliptic problems. EPFL, MATHICSE Report 34, submitted, 2012.

16. P. Chen, A. Quarteroni, and G. Rozza. Stochastic optimal Robin boundary control problems of advectiondominated elliptic equations. EPFL, MATHICSE Report 23, submitted, 2012.

17. P. Crosetto, S. Deparis, G. Fourestey, and A. Quarteroni. Parallel algorithms for fluid-structure interaction problems in haemodynamics. SIAM Journal on Scientific Computing, 33(4):1598-1622, 2011.

18. C. D'Angelo and A. Quarteroni. On the coupling of $1 \mathrm{~d}$ and $3 \mathrm{~d}$ diffusion-reaction equations. application to tissue perfusion problems. Mathematical Models and Methods in Applied Sciences, 18(8):1481-1504, 2008.

19. A.C. Davison. Statistical models. Cambridge University Press, 2003.

20. S. Deparis, M. Discacciati, G. Fourestey, and A. Quarteroni. Fluid-structure algorithms based on steklov-poincaré operators. Computer Methods in Applied Mechanics and Engineering, 195(41-43):5797-5812, 2006.

21. G.S. Fishman. Monte Carlo: Concepts, Algorithms, and Applications. Springer, 1996.

22. L. Formaggia, D. Lamponi, and A. Quarteroni. One-dimensional models for blood flow in arteries. Journal of engineering mathematics, 47(3):251-276, 2003.

23. L. Formaggia, A. Quarteroni, and A. Veneziani. Cardiovascular Mathematics: Modeling and simulation of the circulatory system, volume 1. Springer, 2009.

24. L. Grinberg, T. Anor, JR Madsen, A. Yakhot, and GE Karniadakis. Large-scale simulation of the human arterial tree. Clinical and Experimental Pharmacology and Physiology, 36(2):194-205, 2009.

25. A. Klimke. Uncertainty modeling using fuzzy arithmetic and sparse grids. Universität Stuttgart. PhD thesis, Universität Stuttgart, 2006.

26. D. Kressner and C. Tobler. Low-rank tensor Krylov subspace methods for parametrized linear systems. SIAM Journal on Matrix Analysis and Applications, 32(4):1288-1316, 2011.

27. T. Lassila, A. Manzoni, A. Quarteroni, and G. Rozza. A reduced computational and geometrical framework for inverse problems in haemodynamics. MATHICSE Report 12, 2011.

28. A.C.I. Malossi. Partitioned Solution of Geometrical Multiscale Problems for the Cardiovascular System. PhD thesis, 2012.

29. A.C.I. Malossi, P.J. Blanco, and S. Deparis. A two-level time step technique for the partitioned solution of onedimensional arterial networks. Computer Methods in Applied Mechanics and Engineering, 237-240(0):212-226, 2012.

30. F. Nobile. Coupling strategies for the numerical simulation of blood flow in deformable arteries by $3 \mathrm{~d}$ and $1 \mathrm{~d}$ models. Mathematical and Computer Modelling, 49(11-12):2152-2160, 2009.

31. F. Nobile, R. Tempone, and C.G. Webster. An anisotropic sparse grid stochastic collocation method for partial differential equations with random input data. SIAM Journal on Numerical Analysis, 46(5):2411-2442, 2008.

32. F. Nobile, R. Tempone, and C.G. Webster. A sparse grid stochastic collocation method for partial differential equations with random input data. SIAM Journal on Numerical Analysis, 46(5):2309-2345, 2008.

33. A. Nouy. A generalized spectral decomposition technique to solve a class of linear stochastic partial differential equations. Computer Methods in Applied Mechanics and Engineering, 196(45-48):4521-4537, 2007.

34. K. Ouriel. Peripheral arterial disease. The lancet, 358(9289):1257-1264, 2001.

35. A. Quaini and A. Quarteroni. A semi-implicit approach for fluid-structure interaction based on an algebraic fractional step method. Mathematical models and methods in applied sciences, 17(06):957-983, 2007. 
36. A. Quarteroni and L. Formaggia. Mathematical modelling and numerical simulation of the cardiovascular system. Handbook of numerical analysis, 12:3-127, 2004.

37. A. Quarteroni, R. Sacco, and F. Saleri. Numerical Mathematics. Springer, 2007.

38. A. Quarteroni, M. Tuveri, and A. Veneziani. Computational vascular fluid dynamics: problems, models and methods. Computing and Visualization in Science, MS\&A Series, 2(4):163-197, 2000.

39. P. Reymond, F. Merenda, F. Perren, D. Rüfenacht, and N. Stergiopulos. Validation of a one-dimensional model of the systemic arterial tree. American Journal of Physiology-Heart and Circulatory Physiology, 297(1):H208-H222, 2009.

40. G. Rozza. Shape design by optimal flow control and reduced basis techniques: Applications to bypass configurations in haemodynamics. PhD thesis, EPFL, 2005.

41. A. Saltelli, K. Chan, E.M. Scott, et al. Sensitivity analysis, volume 134. Wiley New York, 2000.

42. A. Saltelli, M. Ratto, T. Andres, F. Campolongo, J. Cariboni, D. Gatelli, M. Saisana, and S. Tarantola. Global sensitivity analysis: the primer. Wiley Online Library, 2008.

43. S. Sankaran and A.L. Marsden. A stochastic collocation method for uncertainty quantification and propagation in cardiovascular simulations. Journal of Biomechanical Engineering, 133(31):228-240, 2011.

44. S.J. Sherwin, L. Formaggia, J. Peiro, and V. Franke. Computational modelling of 1d blood flow with variable mechanical properties and its application to the simulation of wave propagation in the human arterial system. International Journal for Numerical Methods in Fluids, 43(6-7):673-700, 2003.

45. S.A. Smolyak. Quadrature and interpolation formulas for tensor products of certain classes of functions. In Doklady Akademii Nauk SSSR, volume 4, pages 240-243, 1963.

46. C. Soize. Construction of probability distributions in high dimension using the maximum entropy principle: Applications to stochastic processes, random fields and random matrices. International Journal for Numerical Methods in Engineering, 76(10):1583-1611, 2008.

47. N. Westerhof, N. Stergiopulos, and M. Noble. Snapshots of hemodynamics: an aid for clinical research and graduate education. Springer, 2010.

48. D. Xiu and J.S. Hesthaven. High-order collocation methods for differential equations with random inputs. SIAM Journal on Scientific Computing, 27(3):1118-1139, 2005.

49. D. Xiu and S.J. Sherwin. Parametric uncertainty analysis of pulse wave propagation in a model of a human arterial network. Journal of Computational Physics, 226(2):1385-1407, 2007.

50. P. Zunino. Mathematical and numerical modeling of mass transfer in the vascular system. PhD thesis, EPFL, 2002. 